\title{
Automatic and robust noise suppression in EEG and MEG : The SOUND algorithm
}

\section{Mutanen, Tuomas $\mathrm{P}$.}

2018-02-01

Mutanen, T P , Metsomaa , J , Liljander , S \& Ilmoniemi , R J 2018 , ' Automatic and robust noise suppression in EEG and MEG : The SOUND algorithm ', Neurolmage , vol. 166 , pp. 135-151 . https://doi.org/10.1016/j.neuroimage.2017.10.021

http://hdl.handle.net/10138/298238

https://doi.org/10.1016/j.neuroimage.2017.10.021

publishedVersion

Downloaded from Helda, University of Helsinki institutional repository.

This is an electronic reprint of the original article.

This reprint may differ from the original in pagination and typographic detail.

Please cite the original version. 


\title{
Automatic and robust noise suppression in EEG and MEG: The SOUND algorithm
}

\author{
Tuomas P. Mutanen ${ }^{\mathrm{a}, \mathrm{b}, *, 1}$, Johanna Metsomaa ${ }^{\mathrm{a}, \mathrm{b}, 1}$, Sara Liljander ${ }^{\mathrm{a}, \mathrm{c}}$, Risto J. Ilmoniemi ${ }^{\mathrm{a}, \mathrm{b}}$ \\ ${ }^{\text {a }}$ Department of Neuroscience and Biomedical Engineering, Aalto University School of Science, P.O. Box 12200, FI-00076, AALTO, Finland \\ b BioMag Laboratory, HUS Medical Imaging Center, Helsinki University Hospital, P.O. Box 340, FI-00029, HUS, Finland \\ ${ }^{\mathrm{c}}$ Department of Clinical Neurophysiology, Jorvi Hospital, HUS Medical Imaging Center, Helsinki University Central Hospital, P.O. Box 800, FI-00029, HUS, Finland
}

\section{A R T I C L E I N F O}

\section{Keywords:}

Electroencephalography

Magnetoencephalography

Artifacts

Noise

Wiener estimation

Cross-validation

Minimum-norm estimation

\begin{abstract}
A B S T R A C T
Electroencephalography (EEG) and magnetoencephalography (MEG) often suffer from noise- and artifactcontaminated channels and trials. Conventionally, EEG and MEG data are inspected visually and cleaned accordingly, e.g., by identifying and rejecting the so-called "bad" channels. This approach has several shortcomings: data inspection is laborious, the rejection criteria are subjective, and the process does not fully utilize all the information in the collected data.

Here, we present noise-cleaning methods based on modeling the multi-sensor and multi-trial data. These approaches offer objective, automatic, and robust removal of noise and disturbances by taking into account the sensor- or trial-specific signal-to-noise ratios.

We introduce a method called the source-estimate-utilizing noise-discarding algorithm (the SOUND algorithm). SOUND employs anatomical information of the head to cross-validate the data between the sensors. As a result, we are able to identify and suppress noise and artifacts in EEG and MEG. Furthermore, we discuss the theoretical background of SOUND and show that it is a special case of the well-known Wiener estimators. We explain how a completely data-driven Wiener estimator (DDWiener) can be used when no anatomical information is available. DDWiener is easily applicable to any linear multivariate problem; as a demonstrative example, we show how DDWiener can be utilized when estimating event-related EEG/MEG responses.

We validated the performance of SOUND with simulations and by applying SOUND to multiple EEG and MEG datasets. SOUND considerably improved the data quality, exceeding the performance of the widely used channelrejection and interpolation scheme. SOUND also helped in localizing the underlying neural activity by preventing noise from contaminating the source estimates. SOUND can be used to detect and reject noise in functional brain data, enabling improved identification of active brain areas.
\end{abstract}

\section{Introduction}

Electroencephalography (EEG) and magnetoencephalography (MEG) are non-invasive functional imaging methods that are able to record postsynaptic neural activity with excellent temporal resolution. Because MEG and EEG sensors are very sensitive, they are highly susceptible to noise contamination (Ferree et al., 2001; Vrba and Robinson, 2001), which may lead to erroneous interpretations of the cortical activity. We present an effective way to clean noisy EEG/MEG signals by optimally utilizing the multi-sensor data with the help of Wiener estimation. The suggested technique makes use of the bioelectromagnetic model of the head to distinguish noise from neural signals.
Common approaches to deal with noisy data are frequency-domain filtering and the visual identification and rejection of poor-quality sensors or data segments (e.g., Styliadis et al., 2014; Van der Meer et al., 2013; Tewarie et al., 2014; Dominguez et al., 2014). However, the frequency range of the noise may not have well-specified limits, or the noise spectra and the neural spectra of interest may overlap (Herrmann and Demiralp, 2005; Jensen and Colgin, 2007; Monto et al., 2008). Second, visual inspection of the data is laborious, and making rejection decisions is highly subjective. Finally, rejecting sensors always reduces the data dimensionality (amount of information), which cannot be regained even if interpolation is used to reconstruct the data.

We present an objective and robust methodology to quantify noise

\footnotetext{
* Corresponding author. Aalto University School of Science, Department of Neuroscience and Biomedical Engineering, Espoo, P.O. Box 12200, FI-00076, AALTO, Finland.

E-mail address: tuomas.mutanen@aalto.fi (T.P. Mutanen).

1 These authors have contributed equally to this work.
} 
levels in different parts of the data and to correct the measured signals in a way that optimally utilizes the gathered multidimensional information. We call this approach the source-estimate-utilizing noise-discarding algorithm (the SOUND algorithm, hereafter referred to as SOUND). SOUND detects noise automatically by assessing, with the help of a forward model, how well the signal in each channel can be predicted from the rest of the signals. Each sensor is then corrected based on the signal-to-noiseratio (SNR) values of all the recording sensors.

We show that SOUND is a special case of a more general class of Wiener estimators, which minimize the mean-squared error in estimating the noiseless signal. In addition to SOUND, an alternative Wienerestimation approach, which can be computed based only on the recorded data samples, is discussed. In this paper, we refer to this general technique as data-driven Wiener (DDWiener). By applying the DDWiener approach to event-related responses, we illustrate how the noise in individual trials can be automatically detected and taken into account so that the SNR of the estimated evoked response is maximized. The presented data-correction methods work automatically and without completely rejecting any dimension in the data.

For MEG analysis, powerful noise-cleaning techniques that utilize physical principles to separate noise from the multi-sensor signals, e.g., signal-space separation (SSS) (Taulu et al., 2004; Taulu and Kajola, 2005) and the generalized side lobe canceller (Mosher et al., 2009), have been taken into use. Another common approach in MEG is to measure the environmental noise signals from an empty MEG room prior to subject entering the recording space. The noisy signal-space directions can then be rejected from the actual data, e.g., by using signal-space projection (SSP) (Uusitalo and Ilmoniemi, 1997).

SSP can be used to clean certain EEG artifacts (Mäki and Ilmoniemi, 2011; Mutanen et al., 2016), but it is often difficult to determine the poor-quality signal directions as the empty-room measurement is not possible. In practice, the most common approach to tackle the sensorspecific noise is to identify and reject the noisy channels and to interpolate the data in the rejected channels using, e.g., the spherical-spline basis functions (Perrin et al., 1989) or source modeling (Mutanen et al., 2016; Nieminen et al., 2016). One may also build a surrogate model that describes the brain-derived and the artifactual EEG simultaneously to clean the data (Litvak et al., 2007; Berg and Scherg, 1994); for this purpose, the artifact topographies need to be defined, e.g., with the help of principal component analysis.

If the data contain artifacts that can be characterized by few topographies and the corresponding time-domain samples arise from nonGaussian distributions, independent components analysis (ICA) may also be useful in cleaning the data (Vigário, 1997; Korhonen et al., 2011). Artifactual ICA components are often identified and rejected manually but also some automatic methods have been suggested, e.g., FASTER (Nolan et al., 2010), which uses a set of predefined artifactual features to categorize the obtained ICA components into brain and noise components. However, it is not always true that the noise sources follow the assumptions of ICA.

All the above-mentioned EEG-correction techniques require that the contaminated data are confined to only a few channels or signal-space directions, which are identifiable based on the amplitudes or the distributions of the data samples. It should be noted that these techniques decrease the dimensionality of the data and they may require a notable amount of heuristic knowledge.

Possibly, the algorithm closest to SOUND is Sensor Noise Suppression (SNS) (De Cheveigné and Simon, 2008), which corresponds to DDWiener in the sensor space. As SOUND, SNS detects noise in MEG and EEG channels by comparing the signal in each sensor to the rest of the sensor traces. In SNS, each sensor signal is replaced by that part of the original signal belonging to the signal subspace spanned by the other sensor signals. SNS works well with noise that is completely uncorrelated across different sensors as well as uncorrelated with the brain signal, but it is very sensitive to violations of these assumptions.

As EEG noise cannot be estimated from an empty-room measurement, we here concentrate on studying the performance of SOUND and general Wiener estimation with EEG. We illustrate through sample datasets how SOUND improves EEG signal quality. With simulations, we demonstrate that SOUND improves SNR, is robust against modeling errors, and can improve source localization. We compare the performance of SOUND to the most commonly applied method, the channel rejection and interpolation, as well as to SNS. As a proof of concept, we show that SOUND can also be used to clean evoked MEG data.

\section{Methods}

In this section, we explain the theoretical basis for the methods and describe how we measured, simulated, and analyzed the data.

We use the following notation: an upper-case bold character denotes a matrix, a lower-case bold character denotes a vector, and a non-bolded, italic character refers to a scalar. An element in the ith row and $j$ th column of matrix $\mathbf{X}$ is denoted by $x_{i, j}$. We use the subscript notation $\mathbf{X}_{\cdot . j}$ to indicate that we take the $j$ th column vector of matrix $\mathbf{X}$. To simplify the notation, instead of $\mathbf{x}_{i,}$, we use $\mathbf{x}_{i}$ to indicate the $i$ th row vector of matrix $\mathbf{X}$.

The subscript $\neq i$ means inclusion of all the rows or columns of a matrix/vector except the ith one. Hence, $\mathbf{x}_{\neq i, j}$ is the $j$ th column vector of matrix $\mathbf{X}$ from which the element $x_{i, j}$ has been excluded, $\mathbf{X}_{\neq i}$ refers to a submatrix of $\mathbf{X}$, where the $i$ th row has been excluded, and $\mathbf{X}_{\neq i, \neq j}$ refers to a submatrix of $\mathbf{X}$, where the $i$ th row and the $j$ th column have been excluded.

\section{The SOUND algorithm}

Here, we introduce the SOUND algorithm, which makes use of the multidimensional nature of the data; we estimate the reliability of each sensor, given measurements in all the other sensors, and clean the measured data accordingly.

Both EEG and MEG quantify brain activity by measuring electromagnetic fields created by post-synaptic currents, which form the source currents of the signal. The signals measured by $S$ sensors at $T$ time instants (samples) can be written as

$\mathbf{Y}=\overline{\mathbf{Y}}+\mathbf{N}=\mathbf{L} \mathbf{J}+\mathbf{N}$,

where $\mathbf{Y}$ and $\overline{\mathbf{Y}}$ are $S \times T$ matrices containing the noisy and the noise-free signals, respectively, and $\mathbf{N}$ is an $S \times T$ noise matrix. $\overline{\mathbf{Y}}$ can be expressed as a matrix product between the $S \times J$ lead-field matrix $\mathbf{L}$ and the $J \times T$ source-current matrix $\mathbf{J}, J$ being the number of all the sources. The element $l_{s, j}$ in $\mathbf{L}$ describes the sensitivity of sensor $s$ to source $j$, while $\mathbf{j}_{j}$, the $j$ th row of $\mathbf{J}$, contains the waveform of source $j$.

Our goal is to estimate $\overline{\mathbf{Y}}$ from $\mathbf{Y}$. To achieve this, we first attempt to construct the minimally noisy source estimates, $\widehat{\mathbf{J}}$, and then use $\widehat{\mathbf{J}}$ in reconstructing the cleaned versions of the sensor signals, $\widehat{\mathbf{Y}}$. Note that $\mathbf{J}$ may also include uninteresting brain activity, which generates so-called neural-noise signals. However, our goal here is not to separate the neural-noise component from the data. We wish to minimize the amount of noise and artifacts (of extracranial origin), N, leaking into the source estimate $\widehat{\mathbf{J}}$.

If we knew how the noise is distributed in the sensor space, i.e., the noise covariance matrix $\boldsymbol{\Sigma}$, we should emphasize the most reliable signal directions in the estimation of source currents $\mathbf{J}$. We achieve this by multiplying Eq. (1) from left with $\Sigma^{-1 / 2}$, which corresponds to whitening the data with respect to the noise:

$\left(\boldsymbol{\Sigma}^{-1 / 2}\right) \mathbf{Y}=\left(\boldsymbol{\Sigma}^{-1 / 2}\right) \mathbf{L} \mathbf{J}+\left(\boldsymbol{\Sigma}^{-1 / 2}\right) \mathbf{N}=\tilde{\mathbf{Y}}=\tilde{\mathbf{L}} \mathbf{J}+\tilde{\mathbf{N}}$ 
where $\tilde{\mathbf{Y}}, \tilde{\mathbf{L}}$, and $\tilde{\mathbf{N}}$ are the whitened versions of the signal, lead-field, and noise matrix, respectively. From Eq. (2), J can be estimated as the Tikhonov-regularized minimum-norm estimate $(\mathrm{MNE})^{2}$ :

$\widehat{\mathbf{J}}=\tilde{\mathbf{L}}^{\mathrm{T}}\left(\tilde{\mathbf{L}} \tilde{\mathbf{L}}^{\mathrm{T}}+\lambda \mathbf{I}\right)^{-1} \tilde{\mathbf{Y}}$.

The regularization parameter $\lambda$ can be selected, e.g.,

$\lambda=\lambda_{0} \frac{\operatorname{trace}\left(\tilde{\mathbf{L}} \tilde{\mathbf{L}}^{\mathrm{T}}\right)}{S}$,

where $S$ is the number of sensors and $\lambda_{0}$ is a tuning scalar that can be heuristically chosen, e.g., based on the SNR, $\lambda_{0}=1 /$ SNR (Lin et al., 2006).

To use Eq. (3), we obviously need to know $\boldsymbol{\Sigma}$. If we assume that the noise is uncorrelated across the sensors, the noise covariance matrix becomes diagonal, $\boldsymbol{\Sigma}=\operatorname{diag}\left(\sigma_{1}^{2}, \ldots, \sigma_{S}^{2}\right)$, and it is sufficient to estimate the noise level $\sigma_{s}$ in each sensor $s$. The diagonality assumption simplifies the interpretation of Eqs. (2) and (3); when estimating $\mathbf{J}$, we give more weight to those sensors that have better SNR.

If we knew $\bar{y}_{s, t}$, the noiseless measurement in sensor $s$ at time $t$, then the noise level in $s$ could be computed simply as

$\sigma_{s}=\sqrt{\frac{\sum_{t=1}^{T}\left(y_{s, t}-\bar{y}_{s, t}\right)^{2}}{T}}$.

Provided that we are able to estimate the source currents reliably, we can estimate the noise-free sensor signal $\bar{y}_{s, t}$ by

$\widehat{y}_{s, t}=\mathbf{1}_{s} \widehat{\mathbf{j}}_{, t,}$.

Substituting $\widehat{y}_{s, t}$ to Eq. (5) results in the noise estimate $\widehat{\sigma}_{s}$.

Next, we discuss how Eqs. (2)-(6) can be used to cross-validate the signals measured by different sensors. Let us evaluate the noise level of sensor $s^{\prime}$ using Eq. (5). We look for the most likely value for $\bar{y}_{s^{\prime}, t}$, given the measurements of all the other sensors $\mathbf{y}_{\neq s^{\prime}, t}$. Thus, we first find $\widehat{\mathbf{j}}_{, t,}$ by substituting $\tilde{\mathbf{Y}}=\tilde{\mathbf{Y}}_{\neq s^{\prime}}$ and $\tilde{\mathbf{L}}={\tilde{\mathbf{L}} \neq s^{\prime}}_{s^{\prime}}$ into Eq. (3). We can then estimate the noiseless signal in $s^{\prime}$ by using Eq. (6) and, subsequently, the noise level in the same sensor by using Eq. (5).

We may next continue evaluating the noise level in any other sensor $s^{\prime \prime}$ in an identical way. As we now have quantified the noise level in sensor $s^{\prime}$, we can take this into account by updating $\widehat{\boldsymbol{\Sigma}}$ and whitening the original data again to improve $\widehat{\mathbf{j}}_{, t}$ (Eq. (2) and (3)). From the improved version of $\widehat{\mathbf{j}}_{. t}$, we now estimate the noise in sensor $s^{\prime \prime}$.

The obvious problem is that in order to estimate the noise in sensor $s^{\prime}$, we need to know the noise levels in all the other sensors. We solve this issue by evaluating each sensor several times in an iterative fashion, always updating $\widehat{\boldsymbol{\Sigma}}$ based on the latest noise estimates. Step by step, the noise covariance matrix estimates, and thus, the sensor-signal estimates become more accurate. In the next section, we show a suitable initial guess for $\widehat{\boldsymbol{\Sigma}}$ that can be used to launch the iteration.

To ensure that the suggested cross-validation scheme works properly, EEG data should be referenced to a good-quality channel prior to the iteration. Otherwise, the noise of the chosen reference channel leaks to all the channels, violating the assumption of non-correlated noise. We present an automatic way to choose a suitable reference channel in the next section.

All in all, the noise levels can now be determined by using the following iterative scheme (See also Fig. 1 for a visual illustration of the iteration):

\footnotetext{
${ }^{2}$ When the noise covariance is not known, the MNE operator in Eq. (3) is often used without pre-whitening.
}

1. Re-reference the data to a selected good-quality sensor. Make an initial guess for $\widehat{\boldsymbol{\Sigma}}$ in the chosen reference system.

2. Iterate the estimations of the values $\widehat{\sigma}_{s}, s \in 1,2, \ldots, S$, using Eqs. (2), (3), (5), and (6); at each step a new value for $\widehat{\sigma}_{s}$ is obtained according to

$\widehat{\sigma}_{s}=\sqrt{\frac{\sum_{t=1}^{T}\left(y_{s, t}-\mathbf{1}_{s} \widehat{\mathbf{j}}_{, t}\right)^{2}}{T}}$, where

$\widehat{\mathbf{j}}_{\cdot, t}=\tilde{\mathbf{L}}_{\neq s}^{\mathrm{T}}\left(\tilde{\mathbf{L}}_{\neq s} \tilde{\mathbf{L}}_{\neq s}^{\mathrm{T}}+\lambda \mathbf{I}\right)^{-1} \tilde{\mathbf{y}}_{\neq s, t}$

Update $\widehat{\boldsymbol{\Sigma}}=\operatorname{diag}\left(\widehat{\sigma}_{1}^{2}, \ldots, \widehat{\sigma}_{S}^{2}\right)$ after each iteration and re-whiten the original data.

\section{Repeat step 2 until $\widehat{\boldsymbol{\Sigma}}$ has converged.}

To monitor the convergence of $\widehat{\boldsymbol{\Sigma}}$, we can compute the relative changes in the sensor-specific noise levels between two consecutive full iteration rounds. When the relative change in all the channels is less than a desired threshold, e.g., 1\%, the iteration can be terminated.

We can take the final estimate for the noise covariance matrix and use it to whiten the original data (as in Eq. (2)). As the final step, we use the noise-suppressed source estimates to construct the cleaned versions of the sensor-space signals (Eq. (6)). Thus, the final cleaned version of the data can be written as

$$
\begin{aligned}
& \widehat{\mathbf{Y}}=\mathbf{L}\left(\widehat{\boldsymbol{\Sigma}}^{-1 / 2} \mathbf{L}\right)^{\mathrm{T}}\left(\widehat{\boldsymbol{\Sigma}}^{-1 / 2} \mathbf{L L}^{\mathrm{T}} \widehat{\boldsymbol{\Sigma}}^{-1 / 2}+\lambda \mathbf{I}\right)^{-1} \widehat{\boldsymbol{\Sigma}}^{-1 / 2} \mathbf{Y}, \\
& \lambda=\lambda_{0} \operatorname{trace}\left(\widehat{\boldsymbol{\Sigma}}^{-1 / 2} \mathbf{L L}^{\mathrm{T}} \widehat{\boldsymbol{\Sigma}}^{-1 / 2}\right) / S .
\end{aligned}
$$

\section{Relation between SOUND and Wiener estimation}

In the previous section, we showed how SOUND can be used to estimate noise levels in EEG/MEG channels and to suppress the noise accordingly. SOUND is closely related to Wiener estimation (or Wiener filtering). In this framework, the Wiener filter estimates a single-sensor signal as a linear combination of all the recorded EEG/MEG traces to minimize the mean-squared error (MSE). Assuming in Eq. (1) that the noise has a diagonal covariance matrix and that the sources are independent and identically distributed (i.i.d.), SOUND can be shown to correspond to the Wiener estimator for the noiseless signals, thus providing an optimal solution in the MSE sense (see Appendix A for details).

The Wiener estimator can also be computed based on the measured data samples only, when no information on the underlying head model (lead fields) is available. For this alternative implementation, the noise is again assumed uncorrelated across the sensors, and the sample Wiener estimate, $\widehat{\mathbf{y}}_{s}^{\text {sample }}$, becomes (See Appendix A for the derivation)

$\widehat{\mathbf{y}}_{s}^{\text {sample }}=\mathbf{y}_{s}\left(\mathbf{Y}_{\neq s}\right)^{\mathrm{T}}\left[\mathbf{Y}_{\neq s}\left(\mathbf{Y}_{\neq s}\right)^{\mathrm{T}}\right]^{-1} \mathbf{Y}_{\neq s}$.

To make Eq. (8) more stable, Tikhonov regularization is often used according to (Foster, 1961) as

$\widehat{\mathbf{y}}_{s}^{\text {sample }}(\gamma)=\mathbf{y}_{s}\left(\mathbf{Y}_{\neq s}\right)^{\mathrm{T}}\left[\mathbf{Y}_{\neq s}\left(\mathbf{Y}_{\neq s}\right)^{\mathrm{T}}+\gamma \mathbf{I}\right]^{-1} \mathbf{Y}_{\neq s}$

where $\gamma$ needs to be tuned to a sufficient level to guarantee that the matrix is invertible. In particular, $\gamma$ should be appropriately adjusted when the number of channels approaches or even exceeds the number of samples. Otherwise, the rest of the sensor signals might also explain the noise in sensor $s$. The signals over all the sensors can be corrected by applying Eq. (9) once to each sensor $s$ separately. We here refer to this approach, in general, as the data-driven Wiener estimation method (DDWiener).

In the previous section, we saw that an initial guess for the noise covariance matrix is needed to start SOUND. A natural way is to use 


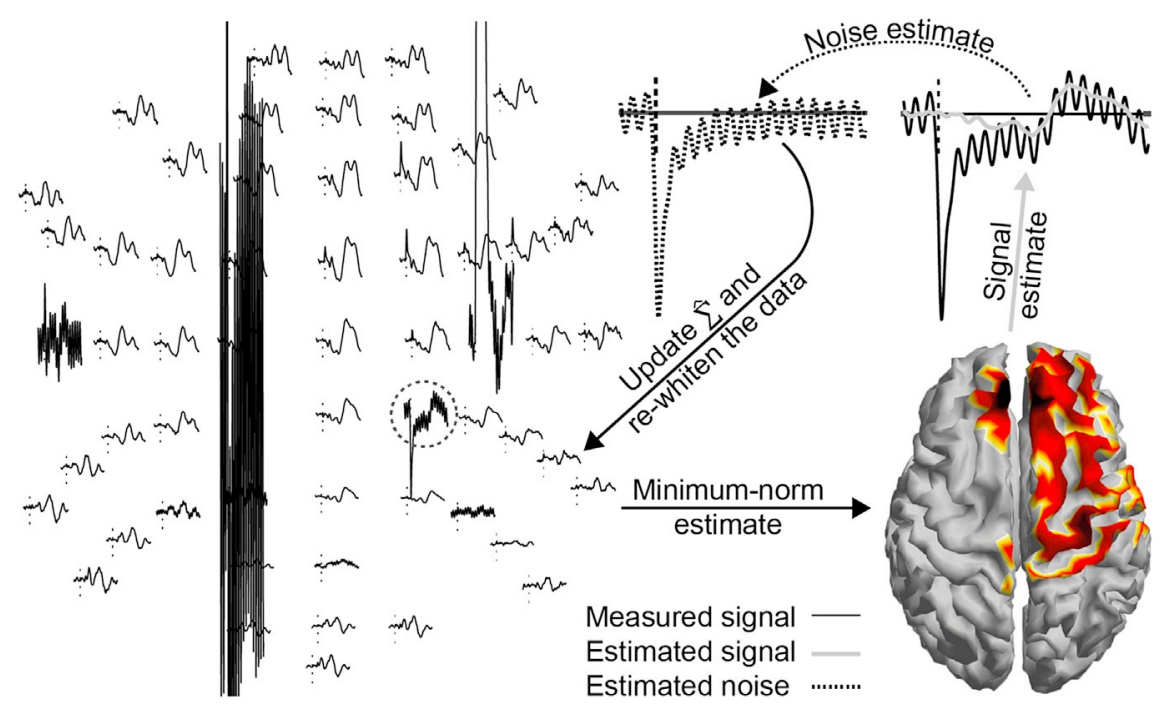

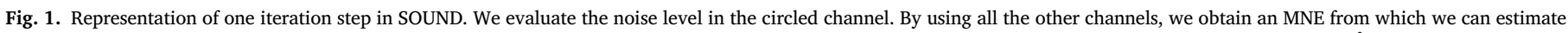

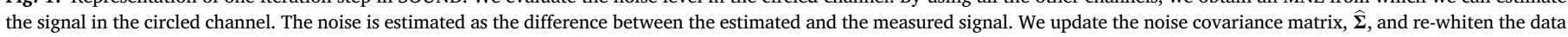
with respect to the most recent noise estimate to improve the accuracy for the next iteration step.

DDWiener to first estimate the noise covariance matrix (Eqs. (9) and (5)). With EEG data, the optimal reference channel with least noise can also be found with DDWiener.

Both Wiener estimators, SOUND and DDWiener, clean signals utilizing the multi-sensor information and assuming diagonal noise structure. The difference between these methods is that SOUND takes additional advantage of the information in the lead-field matrix. Thus, SOUND can be expected to be more robust against noise whose covariance matrix is not perfectly diagonal.

When estimating the noiseless sensor signal with the aid of the other channels, DDWiener corresponds to SNS (De Cheveigné and Simon, 2008) up to the regularization term (Eq. (8) vs. (9), See Appendix A for details). However, as seen in the next section, DDWiener is a general Wiener method that can be applied to multiple problems.

\section{Using Wiener estimation to detect and remove trial-level noise}

The strength of DDWiener (Eq. (9)) is that it can be applied to any multivariate data with a sufficient number of samples. For instance, identifying and rejecting contaminated EEG and MEG trials is still mostly based on visual inspection, although some automatic methods exist (e.g., Jas et al., 2017). Next, to showcase how powerful and flexible Wiener filtering can be in EEG and MEG data analysis, we illustrate how DDWiener can be used to detect noisy EEG or MEG trials. Consequently, the averaged event-related responses can be computed so that the noise contamination is minimized.

We consider event-related data consisting of $R$ trials measured by sensor $s$. The samples are collected into an $R \times T$ data matrix $\mathbf{Y}^{(s)}$, where each row corresponds to the signal in sensor $s$ in one trial. Again, we aim at reconstructing the noiseless signal $\overline{\mathbf{Y}}^{(s)}=\mathbf{Y}^{(s)}-\mathbf{N}^{(s)}$, where $\mathbf{N}^{(s)}$ is the $R \times T$ noise matrix. Making the assumption that the noise is uncorrelated both across the trials and with the noiseless signal, DDWiener can be used in a similar way as in Eq. (9) to get an estimate for the noiseless signal in trial $i$ :

$\widehat{\mathbf{y}}_{i}^{(s)}=\mathbf{y}_{i}^{(s)}\left(\mathbf{Y}_{\neq i}^{(s)}\right)^{\mathrm{T}}\left[\mathbf{Y}_{\neq i}^{(s)}\left(\mathbf{Y}_{\neq i}^{(s)}\right)^{\mathrm{T}}+\gamma \mathbf{I}\right]^{-1} \mathbf{Y}_{\neq i}^{(s)}$

To estimate the noise level in trial $i$ in the sensor $s$, we use
$\sigma_{i}^{(s)}=\sqrt{\frac{\sum_{t=1}^{T}\left(y_{i, t}^{(s)}-\widehat{y}_{i, t}^{(s)}\right)^{2}}{T}}$.

The obtained trial-level noise information can be utilized in subsequent data analysis, e.g., in computing the average evoked response. The averaged EEG response $\mathbf{y}_{\text {ave }}^{(s)}$ in sensor $s$ is

$\mathbf{y}_{\mathrm{ave}}^{(s)}=\frac{\sum_{i} \alpha_{s, i} \mathbf{y}_{i}^{(s)}}{\sum_{i} \alpha_{s, i}}$,

where $\alpha_{s, i}$ is the weight of trial $i$. Conventionally, $\alpha_{s, i}$ gets either the value 1 or 0 depending on whether the trial $i$ is accepted or not, respectively.

If we know how the noise is distributed across the trials, the optimal choice to minimize the power of the noise ${ }^{3}$ in $\mathbf{y}_{\text {ave }}^{(s)}$ is actually

$\alpha_{s, i}=1 /\left(\sigma_{i}^{(s)}\right)^{2}$

Similar weighting has also been suggested by, e.g., Davila and Mobin (1992), Bezerianos et al. (1995), and John et al. (2001) with the distinction that, here, the weights of the trials are obtained by the DDWiener-based noise estimates. The suggested DDWiener methodology is also related to robust averaging (Ashburner et al., 2012), implemented in the SPM toolbox (http://www.fil.ion.ucl.ac.uk/spm/software/spm12/ ), which uses an iterative scheme to find optimal weights in Eq. (12) by evaluating the distance of different samples to the median.

\section{Computation of the lead fields}

When testing SOUND, we used both lead fields based on the subjects' individual anatomy (hereafter referred to as realistic) and simplified lead fields based on a spherical head model.

The realistic lead fields were computed using the boundary element method (BEM) and a conductivity model that included the subjectspecific scalp, inner and outer skull, and gray-matter-white-matter surfaces. For the simulation studies, the source space was discretized in terms of 5120 current dipoles equally spread on the gray-matter-whitematter surface, the dipoles being normal with respect to the surface. For 
cleaning the real-life MEG data with SOUND, the source space consisted of 1640 freely oriented dipoles, while for MEG localization we used an 8200-dipole model. For the TMS-EEG source localization we used a model consisting of 5120 freely oriented dipoles. The relative conductivities of the brain, skull, and skin were $1,1 / 50$, and 1 , respectively. In the simulations, we also tested the sensitivity of SOUND to head-model inaccuracies: we changed the relative skull conductivity to either lower $(1 / 100)$ or higher $(1 / 25)$ than the skull conductivity of the model that was used to simulate the neural signals. The surfaces were based on T1weighted magnetic resonance images of the subject. We used Freesurfer (Fischl, 2012) to segment the gray-matter-white-matter surface and BrainSuite (Shattuck and Leahy, 2002) to segment the scalp and skull surfaces. The lead fields were computed with the combination of the MNE software (Gramfort et al., 2014) and the BEM MATLAB toolbox (Stenroos and Sarvas, 2012). For more details of our segmentation pipeline, see Mutanen et al. (2016).

The concentric spherical head model consisted of the brain, skull, and scalp volume, which had outer radii of 81,85 , and $88 \mathrm{~mm}$, respectively. The relative conductivities were $1,1 / 50$, and 1 , respectively. The lead fields for the spherical head model were computed as explained in Mutanen et al. (2016).

\section{Simulation analysis}

We validated the performance of SOUND by applying it to several simulated EEG sets with various noise characteristics. The simulated data were generated according to Eq. (1). The number of sensors in the simulated data was 60, following a standard 10-20 EEG-electrode system; the number of time points was 146 . The neural data $\overline{\mathbf{Y}}=\mathbf{L J}$ were generated by eight dipolar sources, which were located in the frontal, parietal, temporal, and occipital lobe of the left hemisphere. There were two dipoles in each lobe, one of which was situated superficially on top of a gyrus and the other one about $1 \mathrm{~cm}$ deeper in a sulcus. The waveforms (rows of $\mathbf{J}$ ) and the dipole locations are shown in Fig. 2. The lead field matrix resulting from these dipoles was computed according to a realistic head model, which was constructed based on the MRI of a 26-year-old female who had given her written consent.

The simulated noise was Gaussian and independent of $\overline{\mathbf{Y}}$. The power and the covariance matrix of the noise were adjusted separately. Since the noise covariance matrix of measured EEG/MEG may not perfectly fulfill the diagonality assumption, we investigated how sensitive SOUND is to different degrees of correlated noise. The noise was randomized using 60 independent noise sources. In the simulated EEG data, each noise source was recorded by a fixed number of channels. Here, this number is called the noise correlation index (NCI). When $\mathrm{NCI}=1$, each noise source was measured by one of the channels only, and thus, the noise was completely uncorrelated across the channels. As NCI increased, the amount of correlation increased, the noise being correlated across all the sensors at $\mathrm{NCI}=60$. An additional challenge for the methods was
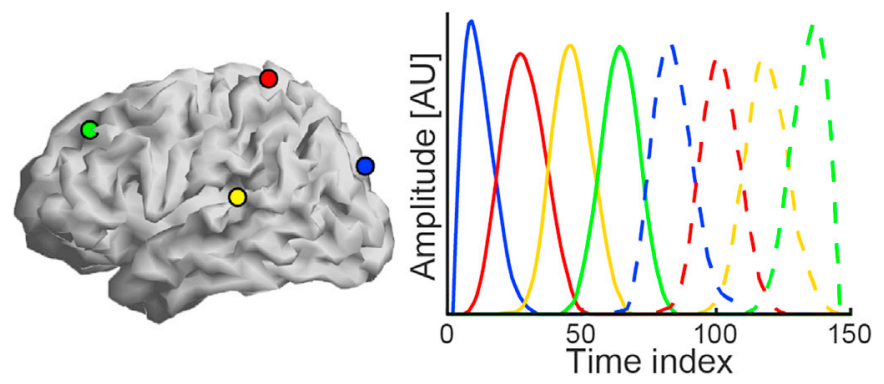

Fig. 2. Simulated neural source locations and the corresponding source waveforms. The colored circles depict the cortical locations of the sources. The dashed curves show the waveforms of the deep sources (about $1 \mathrm{~cm}$ inwards along the sulcus from each circle), whereas the solid curves illustrate the waveforms of the superficial sources located exactly at the circles. that, with increasing NCI, the noise began to resemble the background neural activity in EEG, i.e., the neural noise. The detailed mathematical description of constructing the noise is given in Appendix B.

\section{Evaluating the accuracy of SOUND}

We cleaned the simulated datasets by using SOUND and evaluated its accuracy and robustness as follows.

SOUND was applied according to Section The SOUND algorithm. The reference electrode was chosen to be the channel with the lowest noise level according to Eqs. (9) and (5). An initial guess for the noise covariance matrix in the new reference was then obtained by using again Eqs. (9) and (5). For i.i.d. sources $\operatorname{cov}(\overline{\mathbf{Y}})=\lambda^{-1} \mathbf{L L}^{\mathrm{T}}$. Therefore, the regularization constant in Eq. (3) was set according to $\lambda=\operatorname{trace}\left(\mathbf{L L}^{\mathrm{T}}\right) / \operatorname{trace}\left(T^{-1} \widehat{\mathbf{Y}} \widehat{\mathbf{Y}}^{\mathrm{T}}\right)$, where $\widehat{\mathbf{Y}}$ is the latest estimated noiseless data from the previous SOUND iteration.

To assess the sensitivity of SOUND to modeling errors, we tested four different types of lead-field matrices. Three of the lead-field matrices were based on the same anatomical geometry as was used to simulate the neural sources (we refer to these models as realistic). However, we set the relative skull conductivity to be $1 / 100,1 / 50$, and $1 / 25$ of the brain/scalp conductivity. In the forward model that was used to simulate the neural sources, the corresponding value was $1 / 50$. In addition, we tested the use of a spherical-head-model-based lead-field matrix with the relative skull conductivity $1 / 50$ to assess the importance of geometrical accuracy.

To evaluate the accuracy of noise cancellation of SOUND, the relative noise reduction (rNR) was computed as the relative change in the noise amplitudes between the original and the corrected data:

$\mathrm{rNR}=100 \% \times \sum_{s} \frac{\sigma_{s}-\sigma_{s}^{\mathrm{c}}}{\nu_{s}} / \sum_{s} \frac{\sigma_{s}}{\nu_{s}}$,

where $\sigma_{s}$ and $\sigma_{s}^{\mathrm{c}}$ are the standard deviations of the noise in channel $s$ before and after cleaning, respectively, and $\nu_{s}$ is the standard deviation of the noiseless signal in the same channel. After cleaning, the noise was defined as $\widehat{\mathbf{y}}_{s}-\overline{\mathbf{y}}_{s}$. The rNR parameter is $0 \%$ if none of the noise is removed, whereas it becomes $100 \%$ if the signal is perfectly corrected.

We also quantified how much SOUND helps in source localization. The source estimates were computed by Eq. (3) utilizing the final $\widehat{\boldsymbol{\Sigma}}$ provided by the iteration. To evaluate the accuracies of the source estimates, we quantified the source localization error (SLE) as the weighted average distance of the distributed source estimates with respect to the correct dipole locations by

$\mathrm{SLE}=\frac{1}{8} \sum_{m=1}^{8}\left[\frac{1}{\sum_{n}\left|\widehat{j}_{n, t_{m}}\right|} \sum_{n}\left|\widehat{j}_{n, t_{m}}\right|\left\|\mathbf{r}_{n}-\mathbf{r}_{m}^{\mathrm{d}}\right\|_{2}\right]$,

where $n$ runs over all the cortical locations used in the source estimation, $\mathbf{r}_{m}^{\mathrm{d}}$ is the location of simulated dipole $m$ whose waveform is at its maximum at time $t_{m}, \hat{j}_{n, t_{m}}$ is the estimated dipole amplitude at the location $\mathbf{r}_{n}$ and time $t_{m}$, and $\|\bullet\|_{2}$ is the $L_{2}$ norm. The SLE values were only computed when using the realistic head models.

The simulations were run with several values for NCI (1, 3, and 9) and SNR $(0.33,1,3$, and 9), SNR being defined by

$\mathrm{SNR}=\frac{\operatorname{trace}\left(\overline{\mathbf{Y Y}}^{\mathrm{T}}\right)}{\operatorname{trace}\left(\mathbf{N N}^{\mathrm{T}}\right)}$.

For every SNR and NCI, the simulation was run 100 times, each time with a new noise covariance matrix. The quality measures rNR and SLE were averaged over the 100 simulations.

\section{Comparing SOUND with other methods}

When performing source localization based on EEG/MEG, the Tikhonov-regularized MNE by Eq. (3) is commonly used without pre- 
whitening (e.g., Hauk, 2004; Komssi et al., 2004; Hauk and Pulvermüller, 2004; Moratti and Keil, 2005). Therefore, we compared the SLE values (Eq. (15)) obtained when using SOUND to those obtained with the nonwhitened version of the Tikhonov-regularized estimation. We refer to this approach as the $L_{2}$-regularized MNE. Contrary to SOUND, in $L_{2}$ regularization, the noise levels are assumed to be equal across all the sensors. Because we know the SNR of the simulated data, we can directly use this knowledge to set the regularization parameter in Eqs. (3) and (4) using $\lambda_{0}=1 /$ SNR, as suggested by Lin et al. (2006). The lead-field matrix used in the $L_{2}$-regularized estimation was the same as the one used to simulate the neural EEG signals. This "inverse crime" ${ }^{4}$ was permitted in order to get the best possible $L_{2}$-regularized estimate for the comparison.

When analyzing EEG/MEG data, it is common practice to reject 'bad' channels and replace the original signal with some form of interpolation. We evaluated how SOUND performs as compared to the popular spherical spline interpolation (Perrin et al., 1989), which is generally recommended in the literature (Picton et al., 2000; Michel et al., 2004). The implementation of the interpolation was used as given in Bioelectromagnetism MATLAB Toolbox [http://eeg.sourceforge.net/ bioelectromagnetism.html, 15 September 2017]. In real life, channels can be rejected based on visual inspection or some heuristic, quantitative criteria, see, e.g., the PREP pipeline (Bigdely-Shamlo et al., 2015) or the recently published Autoreject (Jas et al., 2017). As the simulated noise levels were known, here, we rejected a channel if its noise level (standard deviation) was three times larger than the average noise level over all the channels. The signals in the rejected channels were then reconstructed with the spherical spline interpolation using all the remaining channel traces.

Each simulated dataset was corrected by two different approaches: SOUND with the spherical head model, which yields more approximative solutions than the realistic head geometry, and the spherical spline interpolation. The rNR values (Eq. (14)) were computed for both methods and averaged over the interpolated channels.

SOUND was also compared to SNS, which corresponds to DDWiener in the sensor space, up to the regularization term. DDWiener was used as explained in Section Relation between SOUND and Wiener estimation and SNS as described in the original work by De Cheveigné and Simon (2008). As SOUND, SNS and DDWiener were tested with different SNR levels and degrees of correlated noise. After the simulation runs, the performance of SNS and DDWiener were quantified with rNR (Eq. (14)).

\section{Demonstrating the presented methods with measured EEG and MEG data}

We verified the functioning of the DDWiener-based bad-quality-trial detection and SOUND with three different real-life datasets. All the measurements followed the declaration of Helsinki. The EEG measurements were approved by the Coordinating Ethics Committee of the Hospital District of Helsinki and Uusimaa, whereas the MEG measurement was approved by the Research Ethics Committee of Aalto University. For detailed information about the specific instruments, stimulus setup, and recording parameters used in different measurements, please refer to Supplementary material I.

\section{TMS-EEG data}

The first sample dataset consists of transcranial-magnetic-stimulation (TMS)-evoked EEG measured from a male subject (age 25). TMS can activate a region of interest in the cortex by applying a rapidly changing magnetic field to the subject's head. The magnetic pulse induces an electric field strong enough to launch action potentials in the superficial cortex. Because of the strong magnetic pulse, concurrently measured EEG

\footnotetext{
${ }^{4}$ The term inverse crime refers to simulation analysis that does not try to address properly the ill-posed nature of the problem. As a consequence, the obtained results can lead to overly optimistic interpretations. (Kaipio and Somersalo, 2006). For instance, in reality, there are always modeling errors in constructing the lead-field matrix.
}

is prone to suffer from large noise and artifact signals (Ilmoniemi and Kičić, 2010; Ilmoniemi et al., 2015). Here, single-pulse TMS was targeted to the right primary motor cortex, to the representation area of the left abductor pollicis brevis muscle. In total, 100 epochs of single-TMS-pulseevoked EEG were recorded, with a 60-electrode TMS-compatible EEG system (Nexstim Plc., Finland), the bandwidth and sampling frequency being $0-350 \mathrm{~Hz}$ and $1450 \mathrm{~Hz}$, respectively.

\section{$A D-E R P$ data}

The second sample dataset comes from an event-related potential (ERP) study where an Alzheimer's disease (AD) patient (female, age 73 years) received auditory stimuli via headphones. The data were contaminated by muscle and movement artifacts caused by the patient's inability to stay still, resulting in a significant amount of correlated noise. The AD-ERP data consisted of auditory ERP responses evoked by a beep sound (the first beep of a 4-beep train; 40 trains in total). The ERPs were recorded by using a Cognitrace EEG system (eemagine Medical Imaging Solutions GmbH, Germany) with a 64-electrode EEG cap (WaveGuard, eemagine Medical Imaging Solutions GmbH, Germany). The bandwidth of the amplifier (Refa8-64 amplifier, TMS International, The Netherlands) was limited to $0-138 \mathrm{~Hz}$ and the sampling frequency was $512 \mathrm{~Hz}$.

\section{Visual evoked MEG data}

The third sample dataset contains 160 epochs of visual evoked MEG responses. The subject (male, age 29) observed flashes of a checkerboard pattern shown to the right visual field. The studied MEG data contain some gradiometer signals that have rather high noise power if sufficient filtering is not applied to the data. The data were measured with a 306sensor MEG system with 204 planar gradiometers and 102 magnetometers (Elekta Neuromag, Elekta Oy, Finland). The bandwidth of the amplifier was $0.03-330 \mathrm{~Hz}$ and the sampling frequency was $1000 \mathrm{~Hz}$.

\section{Data analysis}

From each of the datasets, we extracted the epochs of interest (100 for TMS-EEG, 40 for AD-ERP data, and 160 for MEG) including the time points between -500 and $500 \mathrm{~ms}$ with respect to the stimulus onset. All epochs were high-pass-filtered from $1 \mathrm{~Hz}$ with a fourth-order Butterworth filter and then baseline-corrected with respect to the time interval $-500 \ldots 0 \mathrm{~ms}$. Next, we applied DDWiener to find the trial-specific noise levels for all the sensors (Eq. (10) and (11)). The trial-specific noise levels were used to find the estimate for the average evoked response in each sensor (Eqs. (12) and (13)).

We further analyzed the interval $-50 \ldots 300 \mathrm{~ms}$ and used SOUND to clean the obtained evoked responses. With the EEG data, we used Eqs. (5) and (8) to find the least noisy sensor and referenced both the data and the lead fields to this sensor. When cleaning the EEG data, we decided to validate the performance of SOUND with the spherical-head-modelbased lead-field matrix to assess whether a fairly simple head model would be sufficient for SOUND to clean EEG. With MEG, it is well known that a single spherical head model is not sufficient to explain neuronal activity from all cortical locations (Crouzeix et al., 1999). Thus, when cleaning MEG signals we used the realistic head model based on the subject's magnetic-resonance images. Before running SOUND, the initial guess for the noise covariance matrix was obtained with Eqs. (5) and (8). When running SOUND, we let the noise-estimation iteration to continue until the relative change in all the channels was less than $1 \%$. To find a suitable regularization parameter (Eqs. (3) and (4)) for SOUND, we tested the values of $\lambda_{0}$ from $10^{-4}$ to $10^{3}$ in ten-fold steps.

To evaluate quantitatively how SOUND affected the measured data, we computed the relative change in the SNR due to SOUND in each sensor: 
$\Delta \mathrm{SNR}_{s}=100 \% \times \frac{\frac{\left\|\widehat{\mathbf{y}}_{s}\right\|_{2}}{\left\|\widehat{\mathbf{y}}_{s}\right\|_{2}}-\frac{\left\|\mathbf{y}_{1}^{1}\right\|_{2}}{\left\|\mathbf{y}_{s}\right\|_{2}}}{\frac{\left\|\mathbf{y}_{s}^{1}\right\|_{2}}{\left\|\mathbf{y}_{s}\right\|_{2}}}$,

where $\mathbf{y}_{s}$ and $\widehat{\mathbf{y}}_{s}$ are the signals of sensor $s$ before and after SOUND, respectively, and the superscript 1 indicates that the signal has been lowpass-filtered from $60 \mathrm{~Hz}$. Because we know that in EEG and MEG neural activity manifests itself mostly at low frequencies (Buzsaki and Draguhn, 2004; Hämäläinen et al., 1993), whereas the non-neural noise has a much broader effective frequency range, we use $\left\|\mathbf{y}_{s}^{1}\right\|_{2} /\left\|\mathbf{y}_{s}\right\|_{2}$ as an approximation for the SNR.

In addition to $\triangle S N R$, we also quantified the amount of overcorrection of SOUND in each sensor $\left(\mathrm{OC}_{\mathrm{s}}\right)$ as the absolute value of the correlation coefficient between the cleaned signal and the estimated noise:

$\mathrm{OC}_{s}=\frac{\left|\widehat{\mathbf{y}}_{s}\left(\mathbf{y}_{s}-\widehat{\mathbf{y}}_{s}\right)^{\mathrm{T}}\right|}{\left\|\widehat{\mathbf{y}}_{s}\right\|_{2}\left\|\mathbf{y}_{s}-\widehat{\mathbf{y}}_{s}\right\|_{2}}$,

where $\mathbf{y}_{\mathbf{s}}$ and $\widehat{\mathbf{y}}_{\mathbf{s}}$ are the original and cleaned signals measured by sensor $s$, respectively. The more SOUND overcorrects the data (removes also signals of interest), the more the cleaned signal will correlate with the estimated noise, i.e., the difference between the original and cleaned signal $\left(\mathbf{y}_{s}-\widehat{\mathbf{y}}_{s}\right)$.

After the quantitative analysis, the data were low-pass-filtered from $100 \mathrm{~Hz}$ with a fourth-order Butterworth filter for visualization.

Finally, we studied whether source localization could benefit from SOUND. For this analysis, we used the MEG and TMS-EEG data since the corresponding magnetic-resonance images of the subjects were available. For both data, we tested whether focal cortical activity could explain some aspects of the measured data by fitting a current dipole to the early EEG/MEG deflections. With the TMS-EEG data, we restricted the analysis to the time interval of 2-20 ms after the TMS onset, as the first $2 \mathrm{~ms}$ are blocked by the TMS-compatible amplifier and it is well known that the TMS-evoked activity can spread from M1 to other brain regions after 20 ms (Ilmoniemi et al., 1997; Komssi et al., 2002). The fitted dipoles were freely oriented and laid on the gray-matter-white-matter surface formed by 5120 equally spread grid points. The localization was done separately at each studied time point; each dipole was fitted to the data by using the method of least squares with no prior knowledge of the noise. The dipole producing the best goodness of fit (GOF) (Kaukoranta et al., 1986) was considered to be the best representation for the neuronal current. The dipole fit was considered reliable if GOF exceeded 0.90. The same source localization was done both to the noisy and cleaned TMS-evoked data. The dipole search was applied to the low-pass-filtered data.

When studying the MEG data, we restricted the localization analyses to the time interval of $0-100 \mathrm{~ms}$ after the visual stimulus, as it has been shown that during this interval the primary visual MEG deflections can be modeled as focal dipolar sources (Portin et al., 1999). Now, the set of fitted dipoles consisted of 8196 freely oriented dipoles, located on the gray-matter-white-matter surface. The source-localization procedure was identical with the TMS-EEG data.

\section{MATLAB demo package}

We have prepared a MATLAB demo package that demonstrates how SOUND and DDWiener can be used in practice together with the openaccess EEG analysis toolbox EEGLAB (Delorme and Makeig, 2004). The demo package was implemented so that it works with open-access data available in the HeadIT EEG repository (Delorme et al., 2011), more precisely with the data measured in the "Auditory Two-Choice Response Task with an Ignored Feature Difference" study [http://headit.ucsd.edu/ studies/9d557882-a236-11e2-9420-0050563f2612, 15 September 2017]. To use the data, one has to accept the HeadIT Data Use Agreement and the applied Terms of Use. The description of the demo package as well as the corresponding download link can be found in Supplementary material II.

\section{Validating SOUND with 12 open-access auditory evoked EEG datasets}

In addition to the three sample datasets presented in this work, we validated the functioning of SOUND with the 12 auditory evoked datasets freely accessible in the HeadIT EEG repository (Delorme et al., 2011). The studied datasets came from the "Auditory Two-Choice Response Task with an Ignored Feature Difference" study [http://headit.ucsd.edu/ studies/9d557882-a236-11e2-9420-0050563f2612, 15 September 2017]. The data analysis of the 12 open-access datasets was based on the MATLAB demo package, provided in Supplementary material II. We compared the performance of SOUND to the spatial DDWiener, as well as to SNS. Because it is impossible to know the ground truth of the true noiseless EEG of the studied datasets, we used four different measures, adapted from (Nolan et al., 2010), to quantify the signal quality before and after the different cleaning methods. For more details about the analysis, see Supplementary material III.

\section{Results}

Results with the simulated data

Among the tested methods (SOUND, SNS, and spherical spline

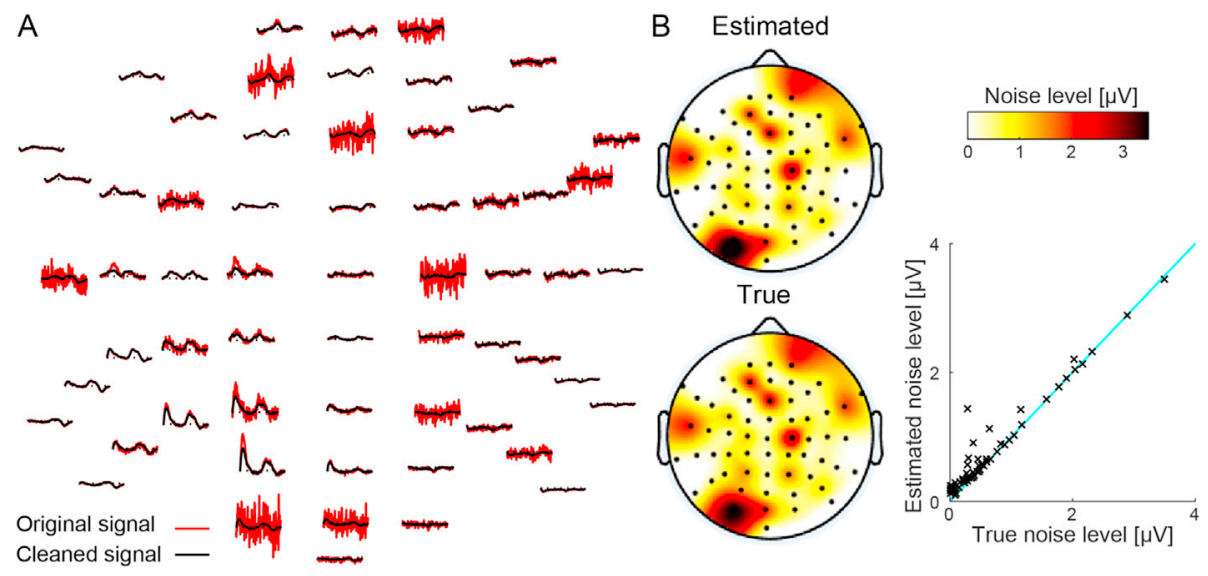

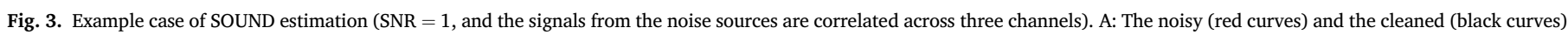

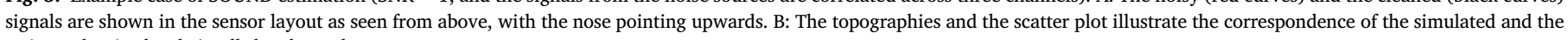
estimated noise levels in all the channels. 
A

The Wiener methods (across all sensors)

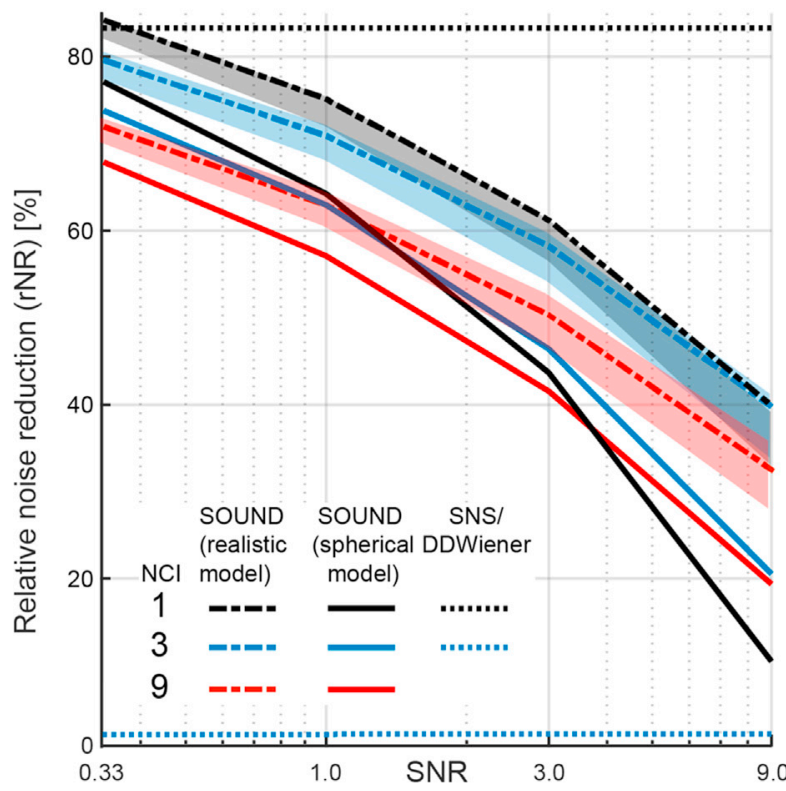

B SOUND vs. Spline interpolation (across rejected sensors only)

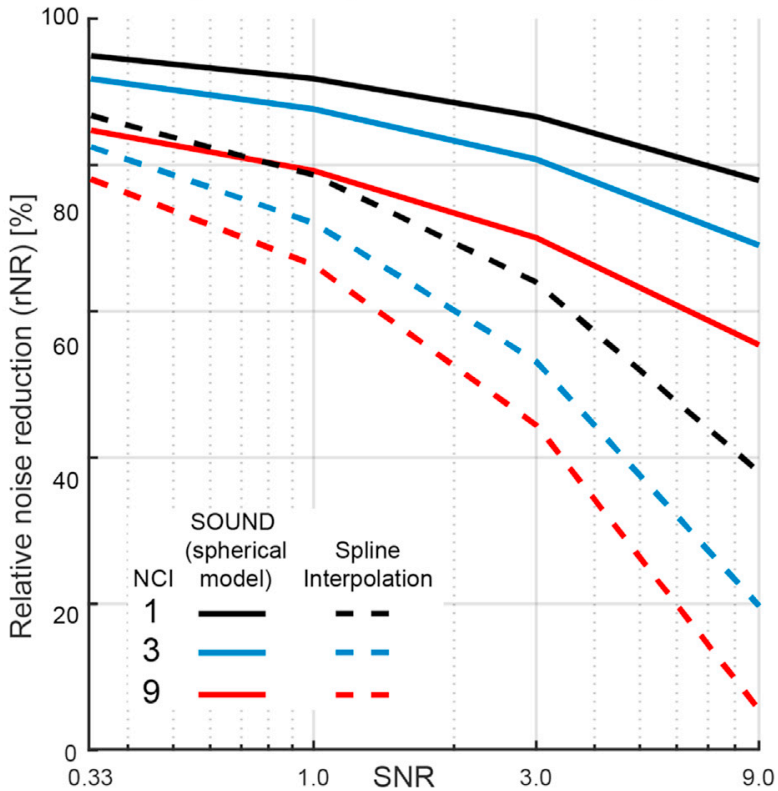

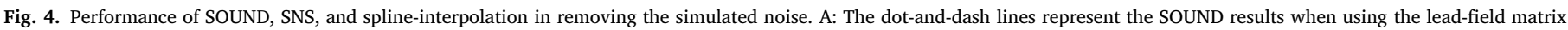

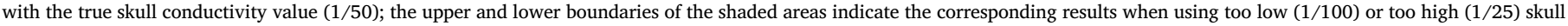

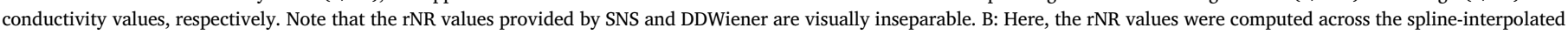
(noisiest) channels only, whereas in the panel A, the rNR values were computed over all the channels.

interpolation), SOUND with the realistic head model was the most robust technique in cleaning the added noise. With an example simulation (with parameters SNR $=1$ and NCI $=3$ ) shown in Fig. 3, we illustrate how contaminated signals were effectively detected and cleaned by SOUND. The true and the estimated noise levels (Fig. 3B) were very well in line, the correlation coefficient being 0.98 . In a few channels, the noise levels were slightly overestimated.

SOUND was robust with respect to small amounts of correlation, which can be seen in Fig. 4A. Moreover, incorrect values of the skull conductivity had only a minor effect on the performance of SOUND. The
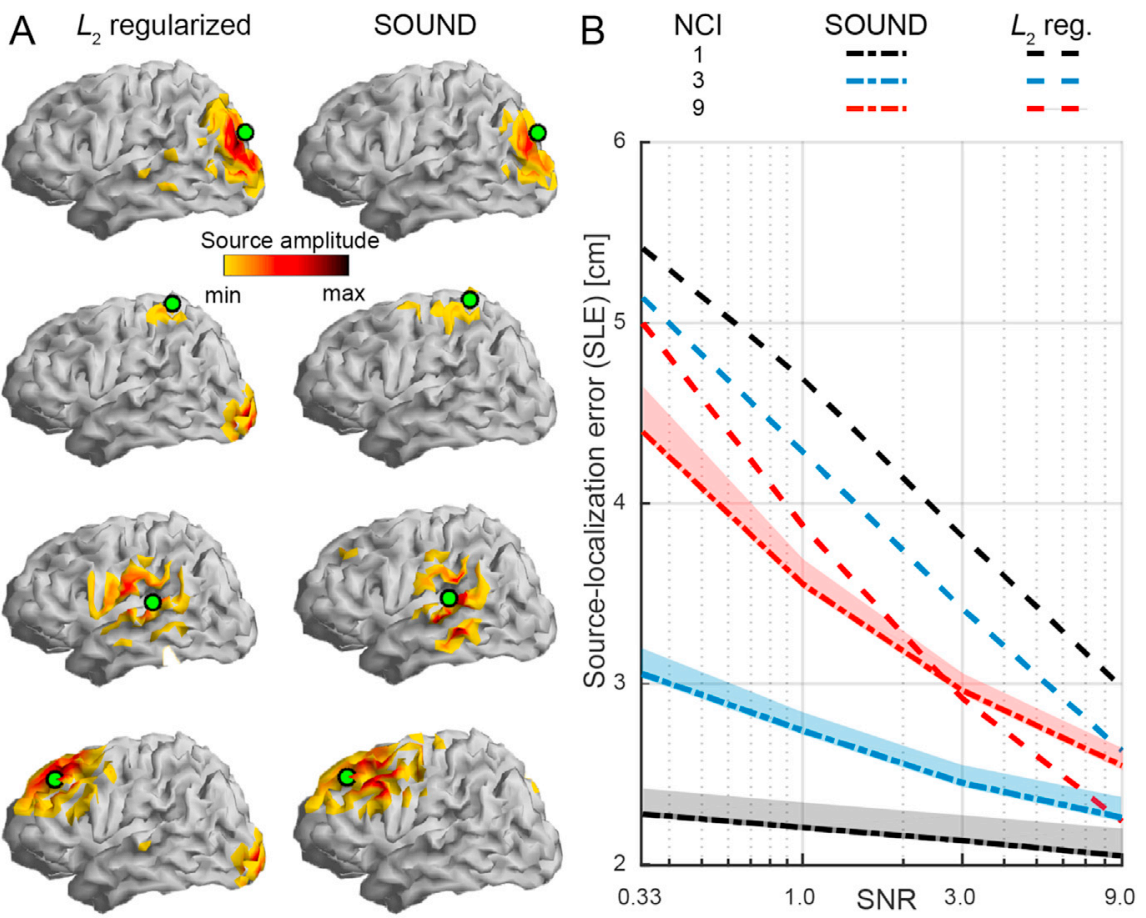

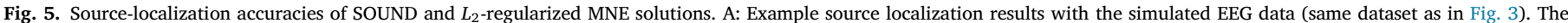

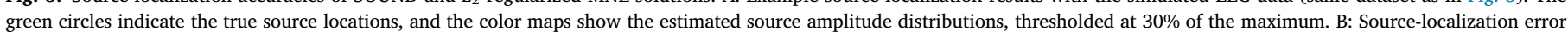

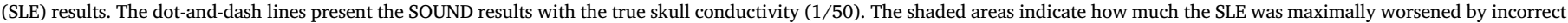
skull conductivities (either too low, i.e., 1/100, or too high, i.e., 1/25). 
A

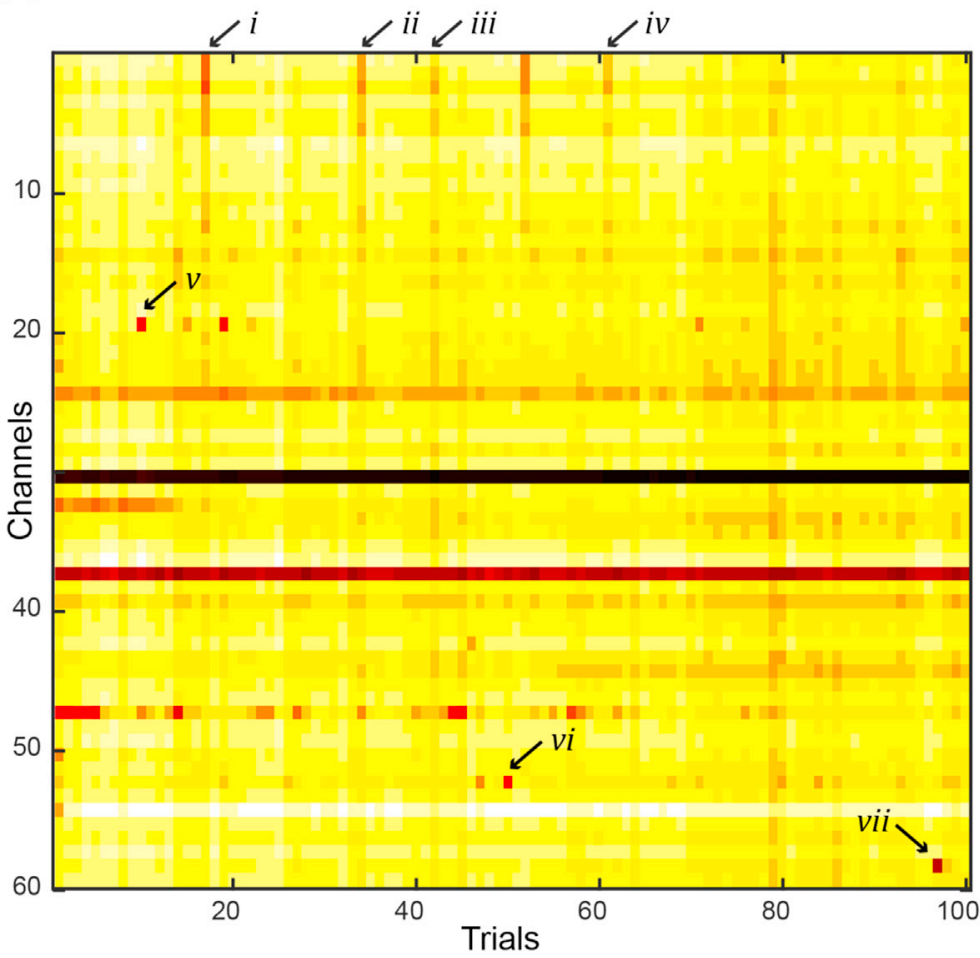

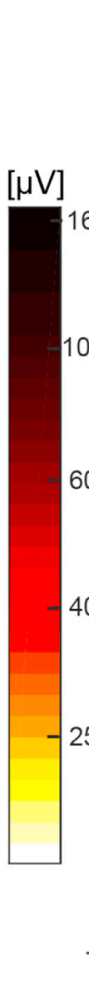

B

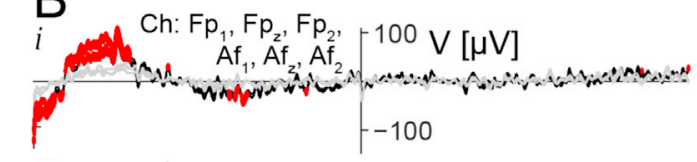

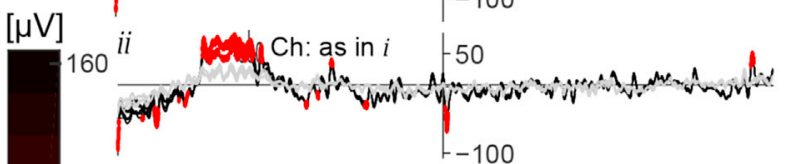

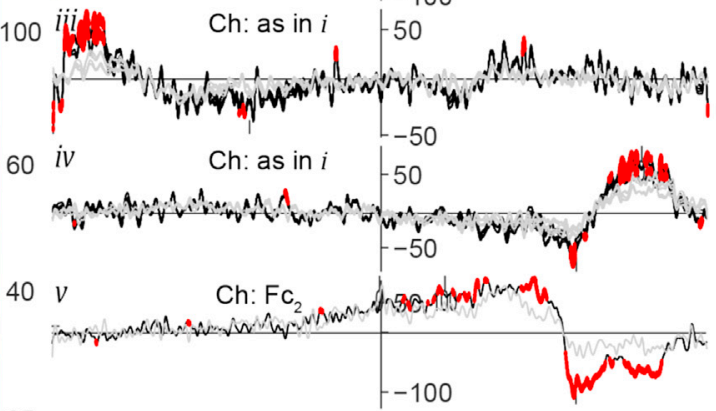

25 vi

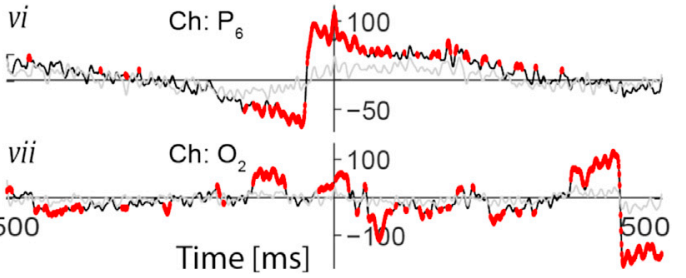

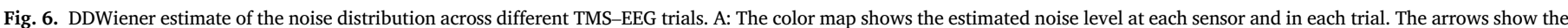

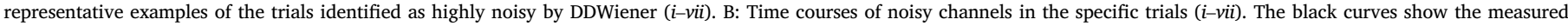

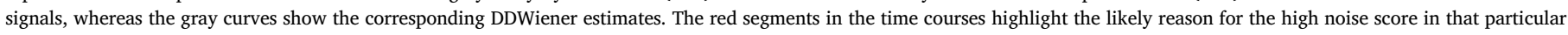
trial, showing the moments when the difference between the measured and estimated signals is greater than in $99 \%$ of all the collected samples.

results of SNS did not depend on the SNR, but in contrast to SOUND, SNS was highly sensitive to the noise correlation structure (Fig. 4A): When the noise was uncorrelated (i.e., NCI $=1$ ), SNS yielded the best rNR among all the tested methods, but even a small amount of correlation over the channels (i.e., $\mathrm{NCI}=3$ ) caused rNR to drop to as low as $2 \%$. As can be expected from the mathematical comparison of SNS and DDWiener (see Appendix A for details), these two methods produced practically identical results, the mean absolute difference in rNR across all conditions being $0.11 \%$ (See Fig. 4A).

SOUND performed better with the realistic head model than with the spherical head model, regardless of the tested simulation parameters or skull conductivity (Fig. 4A). However, the difference between the realistic and spherical head models was considerably smaller when the SNR of the data was low.

SOUND with the spherical head model was compared against the commonly used spherical spline interpolation (Fig. 4B). The channels to be interpolated were selected as described in Section Comparing SOUND with other methods. About 4-6 channels were rejected and interpolated in each simulation. In comparison to the interpolation, SOUND uncovered the noise-free signals more accurately for all the tested simulation parameters, especially with the high SNR values, as indicated by the rNR results.

The $L_{2}$-regularized source localization showed biasing towards cortical locations below the noisy channels, whereas SOUND was robust with respect to noise (Fig. 5A). The source-localization accuracy of SOUND was better than that of $L_{2}$ regularization for all the tested simulation parameters, except for the highest SNR and NCI values, as illustrated in Fig. 5B. The enhanced accuracy of SOUND compared to $L_{2}$ regularization became more apparent as the SNR and/or the correlation of the noise decreased. Unlike with the $L_{2}$-regularized estimation, decreasing SNR did not seem to worsen notably the source-estimation results by SOUND when the NCI was 1 or 3 . When using SOUND, both too low and too high skull conductivities led to slightly increased source- localization errors, the difference to the correct model being about $2.5 \mathrm{~mm}$ at maximum. SOUND-based source localization was robust against small amounts of correlation in the noise.

Results with the measured EEG and MEG data

DDWiener succeeded well in finding contaminated trials. A

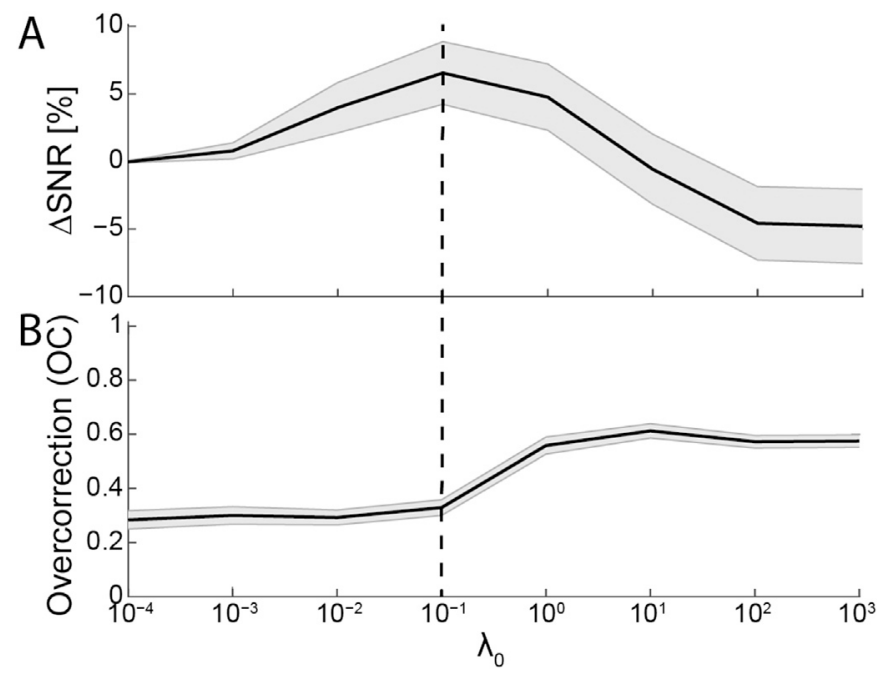

Fig. 7. Performance of SOUND in cleaning the TMS-EEG data with different values of the regularization parameter $\lambda_{0}$. A: SOUND-elicited change in SNR $(\triangle S N R)$ averaged over channels and plotted as a function of $\lambda_{0}$. B: The overcorrection of SOUND (Eq. (18)) shown as a function of $\lambda_{0}$. The dashed vertical line in A and B indicates the choice for $\lambda_{0}$, which provides the best compromise between maximizing the SNR and minimizing the overcorrection. 

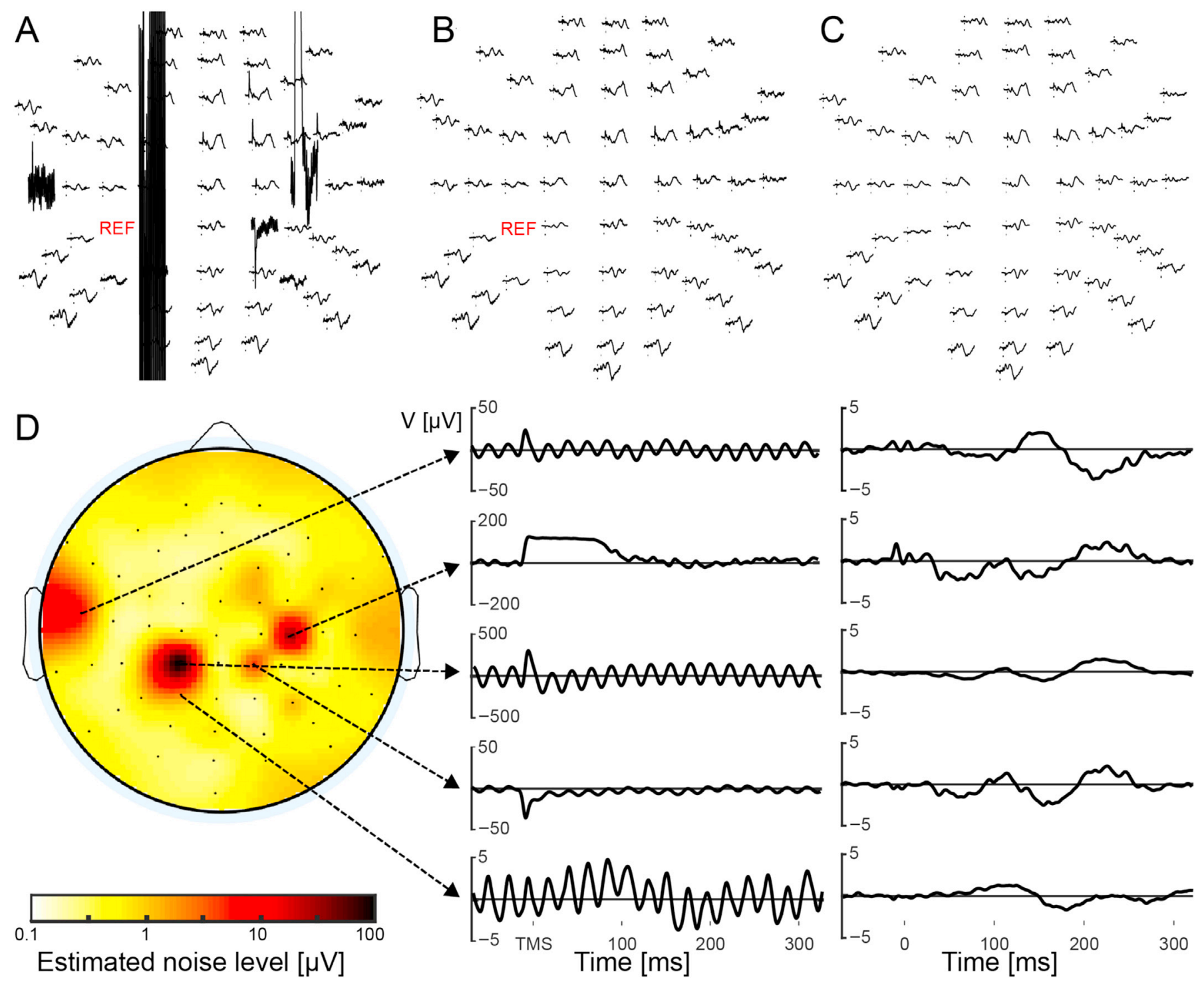

$-5$

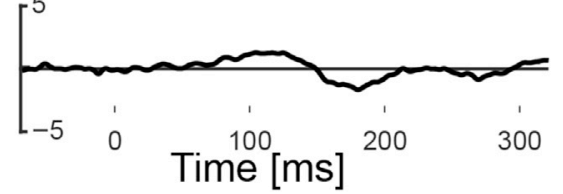

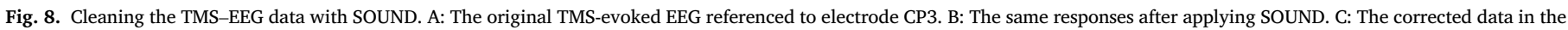

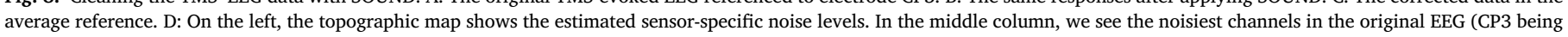
the reference). In the rightmost column, we see the corresponding channels after the SOUND correction.

representative image obtained from the TMS-EEG data is shown in Fig. 6. The visual inspection of the trials showed that DDWiener was sensitive to, e.g., ocular artifacts. Compared to visual inspection, DDWiener was fast in estimating the noise in different trials: evaluation of 100 TMS-EEG, 40 AD-ERP, and 160 MEG trials took 11, 0.7, and $119 \mathrm{~s}$, respectively, with a standard desktop computer.

SOUND was clearly able to improve the signal quality of each of the recorded datasets. The performance of SOUND as a function of the regularization parameter for the TMS-EEG data is shown in Fig. 7. For all the three datasets, the choice of $\lambda_{0}=0.1$ yielded the best compromise between improving SNR and minimizing overcorrection. With the TMS-EEG data, the average $\Delta \mathrm{SNR}_{s}$ was $7 \%$. In total, the SNR among the 36 improved channels increased by $12 \%$, while the corresponding result among the remaining 23 channels was only $-2 \%$. With the choice of $\lambda_{0}=0.1$, SOUND did not seem to overcorrect the data severely, the mean overcorrection index (OC) being 0.33 (the max. value for OC is 1).

With the AD-ERP data, SOUND improved SNR, on average, by $5 \%$; the average increase in the SNR among the 45 improved channels was $8 \%$, whereas the average decrease among the rest 18 channels was $-2 \%$. With the AD-ERP data, OC of SOUND was a bit higher than with the TMS-EEG data, i.e., on average 0.44 .
Because of the very high noise amplitude in several channels, the SNR of the MEG data was improved by $990 \%$, on average. In the 233 improved sensors the mean $\Delta \mathrm{SNR}_{s}$ was $1300 \%$, while the decrease in SNR in the 73 remaining sensors was only $-16 \%$. On average, the OC across the sensors was 0.33 .

Also qualitatively, SOUND performed well. The TMS-evoked responses before SOUND are shown in Fig. 8A, where artifactual and noisy channels are clearly visible. SOUND was able to detect and remove the noise while retaining the clean channels intact (Fig. 8B). SOUND was able to detect several types of defects (e.g., baseline shifts, transient artifacts, and line noise) and correct all the channels successfully, yielding coherent EEG data. Figs. 9A and B visualize through butterfly and topographic plots how SOUND removes the noise and helps to highlight four early TMS-evoked deflections at 4, 17, 27, and $48 \mathrm{~ms}$ after the onset of TMS.

The AD-ERP data were noticeably cleaner after SOUND. Although the muscle artifacts were clearly correlated across the frontal channels, SOUND was able to reduce the noise levels considerably, especially in the most contaminated channels (See Fig. 10). With MEG, SOUND corrected the noisy gradiometers while retaining the signals in the uncontaminated magnetometers. The longitudinal-gradiometer signals are presented 


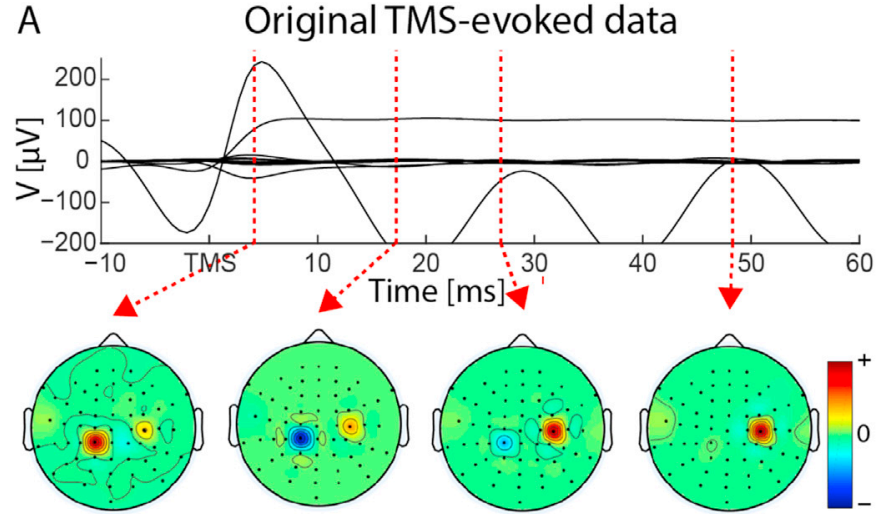

B

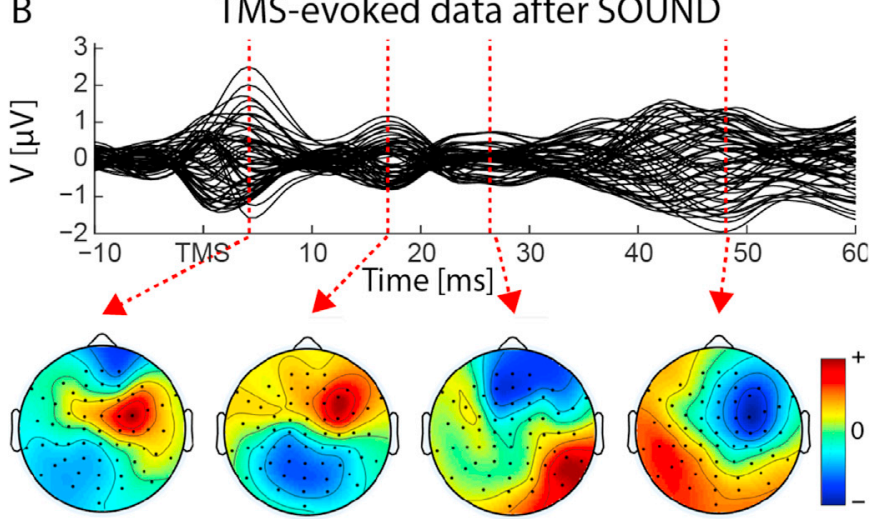

C
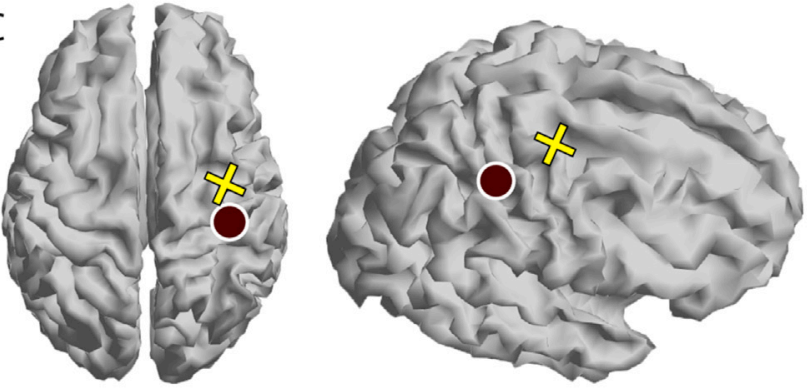

is Stimulus location

Dipole fit

Fig. 9. Effects of SOUND to the TMS-EEG data quality. A: Original data. B: The same data after SOUND. The top panels of (A) and (B) show the butterfly plot of the data before and after cleaning. The vertical red dashed lines in the top panels of (A) and (B) indicate the latencies of the early deflections that were identified from the cleaned data. The topographies illustrate the EEG voltage maps at the identified deflection latencies. C: The location of the best-fitting dipole (GOF > 0.9) at $18 \mathrm{~ms}$ after TMS.

in Fig. 11.

With the assumption that the early TMS-evoked EEG deflections mainly reflect focal cortical activity close to the stimulation target, it seems that SOUND was able to improve the source localization considerably (Fig. 9C). When the dipole search was done with the original TMS-EEG data, no reliable dipole fits were found as the GOF always remained under 0.7. After SOUND, the best-matching dipole was found in the stimulated hemisphere, at $17-20 \mathrm{~ms}$ after the TMS pulse, $2 \mathrm{~cm}$ away from the TMS target (GOF $>0.9$ ).

As with the TMS-EEG data, no reliable dipole fits were found for the MEG data before the SOUND correction (throughout the studied time interval, GOF < 0.78). After SOUND, two dipoles were found in the left visual cortex, at $53-75 \mathrm{~ms}$ and $79-83 \mathrm{~ms}$ after the stimulus presented to the right visual field (GOF $>0.9$ for both dipoles). This is in line with previous findings in the literature (Portin et al., 1999). The dipole fit at $\sim 65 \mathrm{~ms}$ is illustrated in Fig. 11.
When cleaning the EEG sample datasets, five full iteration rounds were enough for SOUND to converge to the requested level. With MEG, 11 iterations were required. With the TMS-EEG, AD-ERP, and MEG data, SOUND fully converged in $\sim 20, \sim 30$, and $\sim 50$ iterations, respectively, after which the noise-estimate changes were merely numeric $\left(\sim 10^{-14} \%\right)$. The changes in the noise-estimates as a function of the iteration step are illustrated in Fig. 12. The resulting cleaned data were practically identical regardless of whether iteration was continued until true convergence or stopped at the suggested 1\%-criterion. For the 60and 64-sensor EEG data, it took less than $5 \mathrm{~s}$ for a standard desktop computer to perform SOUND, whereas with the 306-sensor MEG data the corresponding time was $105 \mathrm{~s}$.

\section{Results with the open-access EEG data}

According to the visual and quantitative analysis, SOUND performed robustly with the open-access datasets. Overall, SOUND was sensitive to different outlier EEG activities, correcting the signals in the contaminated EEG channels to a level that could be expected from neuronal activity. Compared to DDWiener, SOUND was much more efficient in detecting and correcting the outlier activity. Neither DDWiener nor SOUND, seemed to overcorrect the channel signals that more likely reflected intracranial post-synaptic-currents. With these specific datasets, both SOUND and DDWiener clearly outperformed SNS. See Supplementary material II for the detailed description of the quantitative analysis, as well as the corresponding results.

\section{Discussion}

In this work, we introduced methods, most importantly SOUND, to clean EEG/MEG data. SOUND takes a novel approach to identify and separate the neural and noisy data based on their spatiotemporal characteristics. We have provided a theoretical basis for SOUND and tested its functioning under several conditions using both simulated and measured data. Here, we discuss some implications of the obtained results.

\section{Practical considerations on cleaning EEG/MEG signals by Wiener} estimation

Inspecting data visually can be laborious. Since the presented noisecleaning approaches work automatically and require only seconds to few minutes of computational time, they can considerably save the dataprocessing time. Therefore, projects with large populations, e.g., some clinical studies (Bresnahan and Barry, 2002), can significantly benefit from the Wiener estimation methods. In less-cooperative subject groups, such as children or neurological patients, rejecting trials and/or channels completely may lead to an unrepresentative amount of data. Using Wiener estimation would enable the utilization of most of the collected data and prevent valuable recordings from being wasted.

SOUND requires very little user input: once the conductivity model for the head is chosen, SOUND has only one degree of freedom; the regularization parameter may need to be fine-tuned for the measured data. In this work, we showed an example of how a proper value can be found in a data-driven fashion.

When cleaning the visual evoked MEG data with SOUND, we did not take possible head movements into account. If the subject is prone to move inside the MEG helmet, the head movement can be corrected with SOUND using the magnetic-field realignment approach suggested by Numminen et al. (1995) and Uutela et al. (2001).

In this work, SOUND was applied to evoked responses. If the noise is expected to be non-stationary across the trials, SOUND can also be used to clean each trial separately. The cost of this approach is the lengthened computing time. On the other hand, if the noise can be assumed stationary across the trials, but the user wishes to retain the trial-level details, a much faster approach exists; one can estimate the noise 


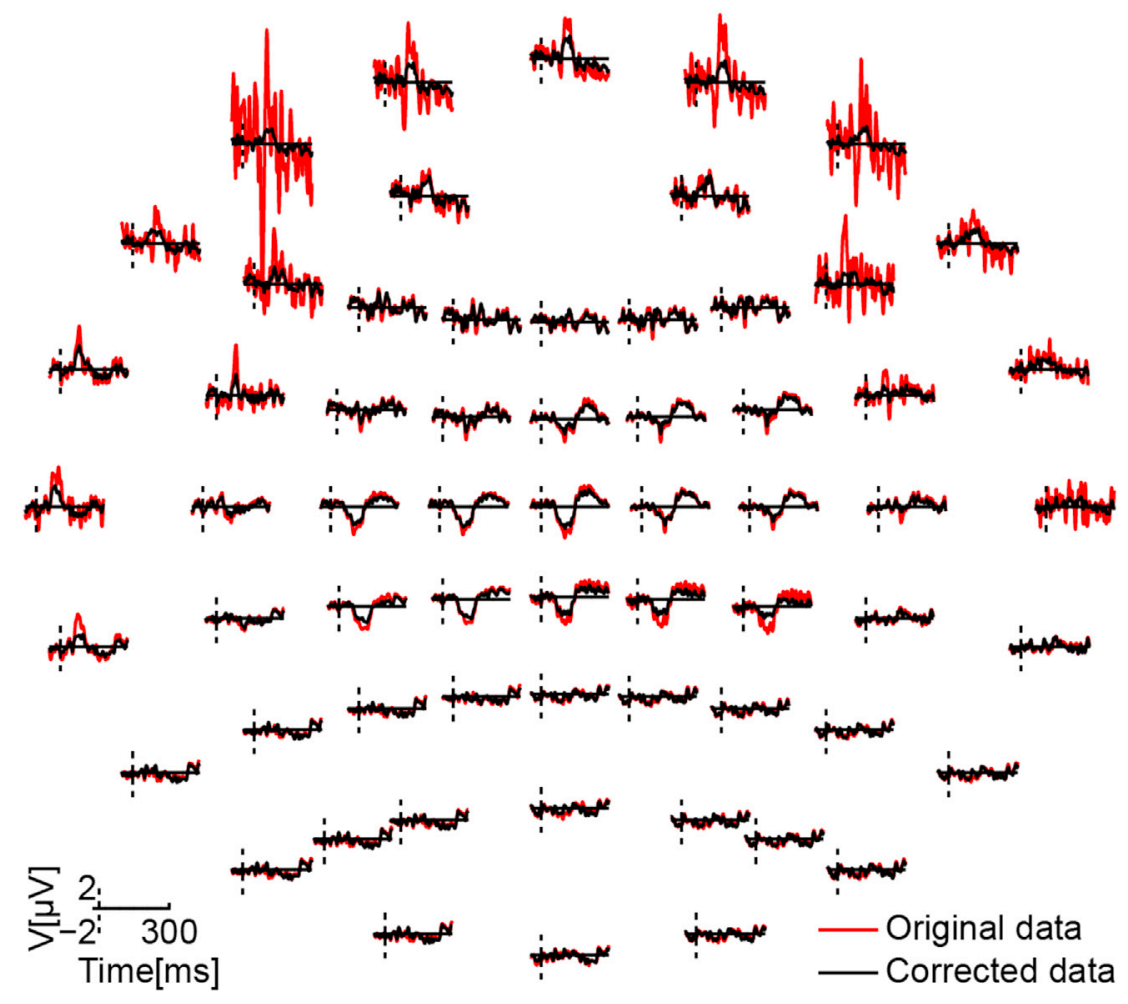

Fig. 10. The AD-ERP data before and after correcting it with SOUND.

covariance matrix from the average data, scale the the obtained noise covariance matrix by multiplying it by the number of trials, and clean each trial accordingly using Eq. (7).

\section{SOUND as a preprocessing step for various types of EEG/MEG analysis}

In the simulations, SOUND performed at least as well as the combined channel-rejection and interpolation scheme. As using SOUND is faster and requires much less heuristic knowledge than the visual inspection of the channels, SOUND is a good candidate to clean contaminated sensors in the preprocessing stage.

SOUND is able to preserve the rank of the data even if the dimensionality of the noise is equal to the number of the sensors. Using several other noise cancellation techniques, e.g., ICA or SSP, removing noise that is present in all the signal-space directions would completely flatten the measured signals. Because SOUND retains the rank, many data analysis methods are applicable subsequent to SOUND.

A considerable amount of EEG and MEG research is based on the sensor-space analysis (e.g., Hong et al. (2004); Ferreri et al. (2011)). If only a few sensors cover the region of interest, even a small number of rejected channels can be highly problematic. SOUND utilizes multisensor recordings and smoothly replaces noisy sensor signals with the best possible estimates.

Measurement noise and the signals of interest can heavily overlap in the frequency domain (Whitham et al., 2007; Goncharova et al., 2003). Because SOUND rejects noise without making any assumptions of the frequency content of the noise, several Fourier-analysis related methods are applicable subsequent to SOUND. For instance, some connectivity measures, such as partial directed coherence (Baccalá and Sameshima, 2001), are computed within a particular frequency range of interest.

In addition to sensor-space analysis, different source localization or blind source separation (BSS) approaches can be useful for interpreting EEG/MEG data. However, these techniques are sensitive to noise (Bertero et al., 1988; Ikeda and Toyama, 2000; Onton et al., 2006). SOUND provides estimates for the noise covariance matrices that can be utilized in many source localization methods, e.g., MNE (Lin et al., 2006) and beamformer techniques (Moiseev et al., 2011). Alternatively, SOUND can be used to preprocess the data for source localization.

There have been several suggestions on how to estimate and utilize the noise covariance matrix in MEG/EEG analysis. It is a common approach to measure the magnetic fields in an empty MEG room and whiten the actual MEG data with respect to the empty-room data (Gramfort et al., 2014; Engemann and Gramfort, 2015; Niso et al., 2016). For EEG, such empty-room recordings are not possible and the noise is often estimated from the baseline data (Parra et al., 2005; Engemann and Gramfort, 2015). For using empty-room or baseline recordings to estimate the noise, we have to assume that the noise will remain the same before and during the actual measurement. In many cases, e.g., with TMS-EEG data, such an assumption is obviously not valid. With SOUND, we estimate the noise covariance matrix directly from the time window of interest utilizing the physical forward model.

Engemann and Gramfort (2015) studied systematically several data-driven approaches to estimate the noise covariance matrix that, unlike SOUND, do not assume diagonal noise structure. Especially those noise-covariance-estimation methods that did not assume diagonality nor constant noise variance across the sensors required a large number of data samples to work properly. On the contrary, SOUND was shown to work with a relatively small number of samples. Note that the estimation of the noise covariance matrix is only an intermediate step of SOUND, the main goal being the cleaning of the contaminated data. If it is possible to accurately estimate the full noise covariance with off-diagonal elements, e.g., by applying some robust covariance-estimation method (Campbell, 1980; Rousseeuw, 1984; Ledoit and Wolf, 2004; Maronna et al., 2006; Khan et al., 2007; Nguyen and Welsch, 2010; Huber, 2011) to a data segment purely consisting of noise, then one can directly use Eq. (7) to clean the data.

Modeling assumptions of SOUND

When using SOUND, we made the following modeling assumptions: 
A

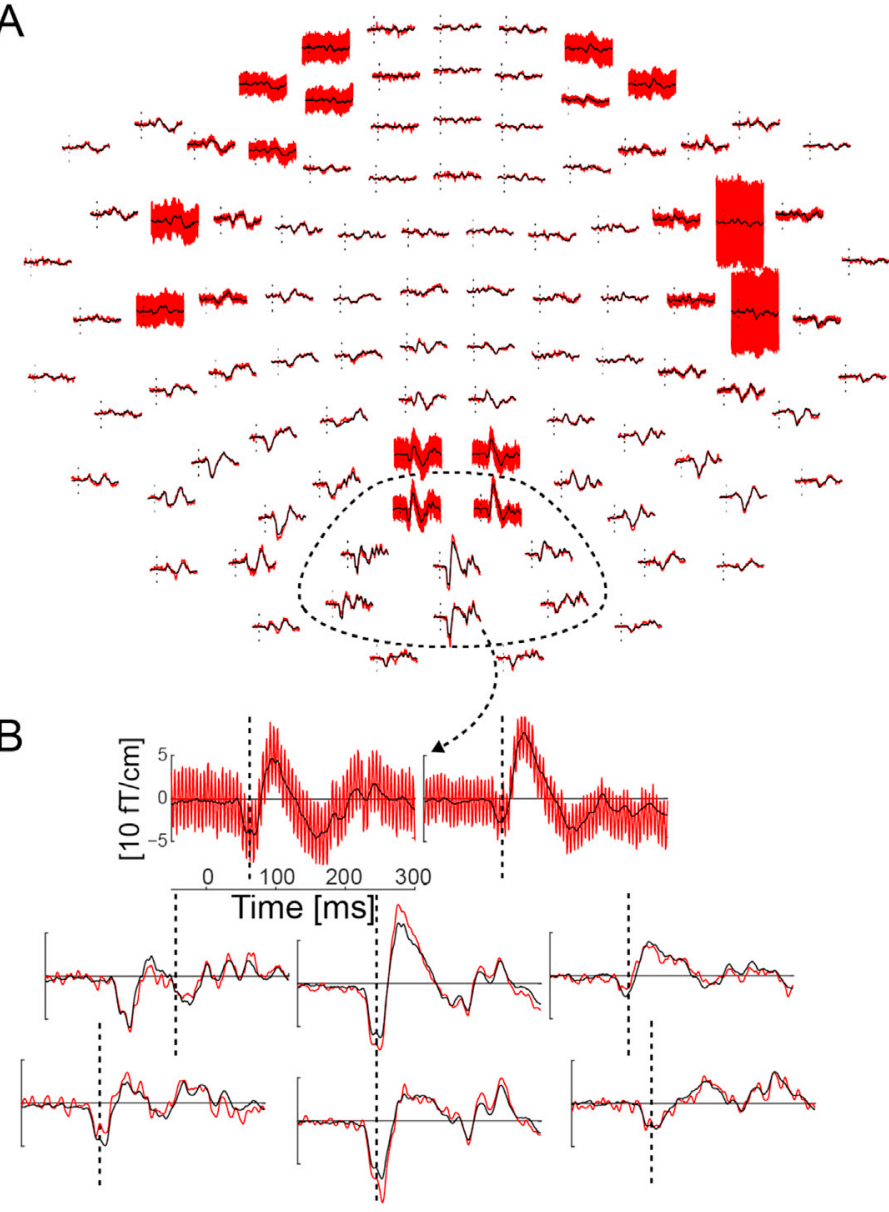

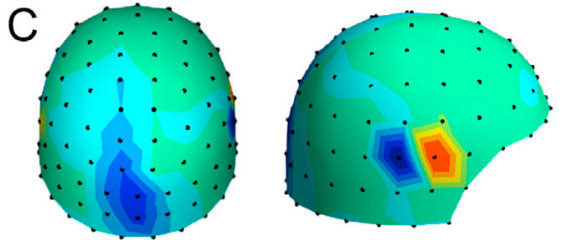
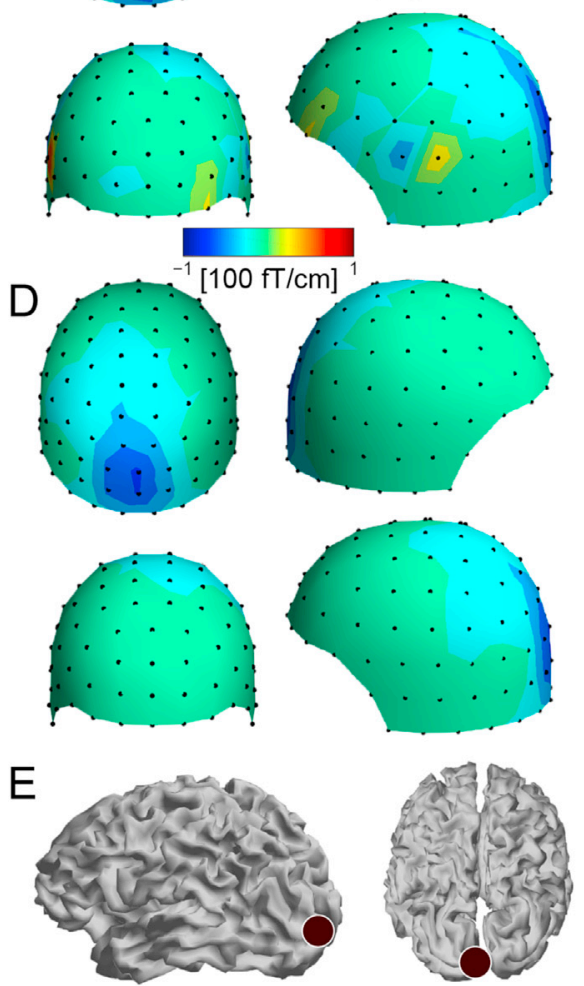

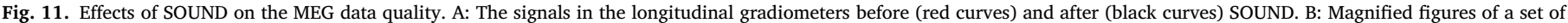

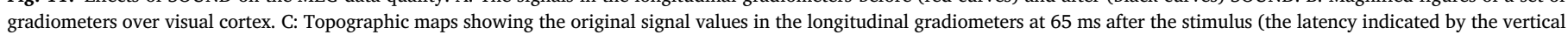
dashed lines in B). D: The corresponding topographies after SOUND. E: The dipole matching best the cleaned signals at 65 ms after the visual stimulus.
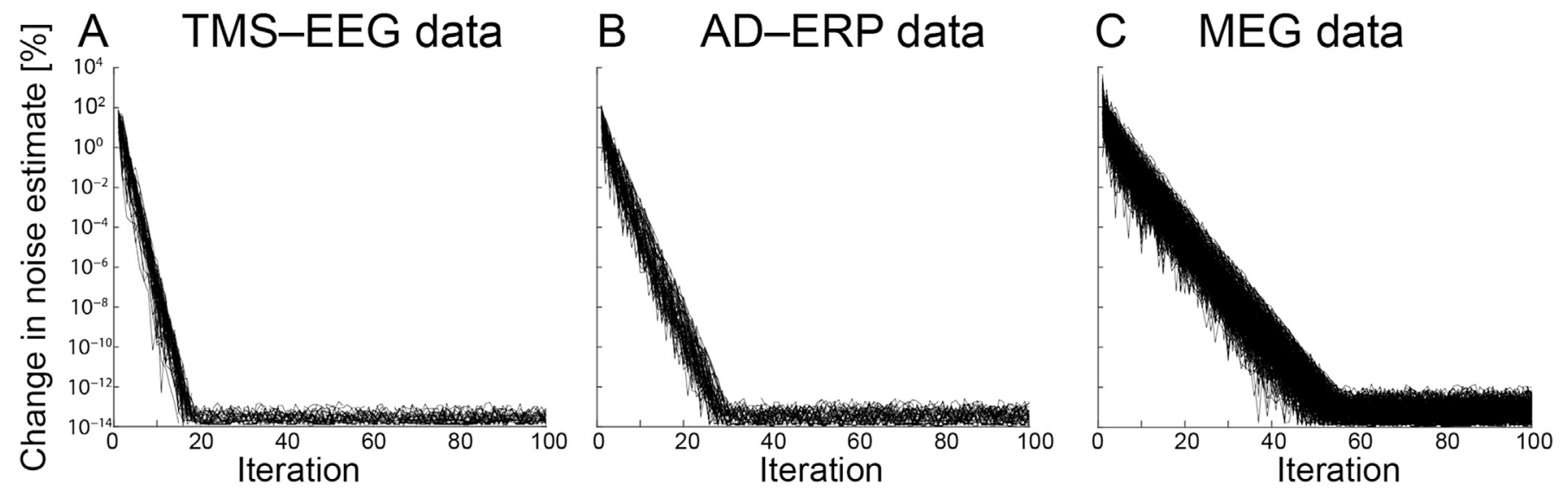

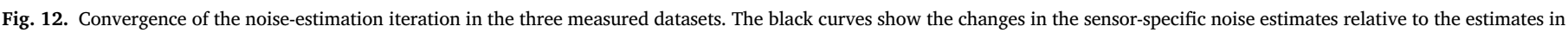
the previous step. The plots show that after few dozens of iterations the changes in the noise estimates are only numeric.

(1) the noise correlation matrix is diagonal, (2) the head conductivity model is known, and (3) the neural sources are i.i.d. The third assumption is not mandatory. If there is prior information on the underlying source distribution, it can be easily included in Eq. (3) in the form of the source covariance matrix (Lin et al., 2006), leading to more accurate data correction. Furthermore, the assumptions (1) and (2) do not need to be fully correct to achieve a good outcome in the noise cancellation.

Unlike with SNS, the noise does not have to be perfectly diagonal for
SOUND to work. This is due to the fact that SOUND also utilizes the information of the neural sources in the form of the lead-field matrix. The simulations also suggest that the conductivity values in the head need to be known only approximately to obtain good data-correction accuracy. The use of realistic geometry was more advantageous compared to the spherical head model. However, also the spherical-head-model-based SOUND proved to work clearly better than the spherical spline interpolation. When using SOUND to clean data with poor SNR, the effects of the 
head-model inaccuracies were less pronounced.

The simulations showed that SOUND may overestimate noise amplitudes in some channels possibly leading to overcorrection. This overcorrection most likely takes place in channels that are not surrounded by a sufficient number of neighboring sensors, or in channels that measure mostly signals from very superficial sources. Indeed, superficial sources generate high-spatial-frequency components in the sensor space, which might be incorrectly identified as noise during the SOUND iteration. In addition, if the used sensor array is much sparser than the arrays studied in this work, it is possible that SOUND attenuates the signals of interest more as the information gathered by different sensors becomes less correlated. The overcorrection might be managed by tuning the amount of regularization. In terms of source localization accuracy, SOUND was beneficial, also with the superficial sources, suggesting that overestimation of noise is not crucial if the focus is mainly on localizing the neural sources.

\section{Comparison of SOUND to existing methods}

In this work, we concentrated in comparing SOUND with the channelrejection and interpolation approach, which is a commonly used method to clean noisy channels (e.g., Julkunen et al. (2011); Casarotto et al. (2013); Atluri et al. (2016)). The channel-rejection-and-interpolation scheme is also used in many signal-processing pipelines and algorithms, such as PREP and Autoreject (Jas et al., 2017). In addition, we compared SOUND to a previously presented method, SNS, which shares several conceptual and theoretical similarities with SOUND.

In the literature, there are suggestions for full EEG and MEG signalprocessing pipelines (Nolan et al., 2010; Gramfort et al., 2014; BigdelyShamlo et al., 2015). Comparing SOUND directly to these pipelines is difficult because SOUND is designed for a specific noise-rejection step, the bad-channel detection and rejection. In the future, one should verify how SOUND could be optimally integrated to some already established EEG/MEG analysis pipelines and/or analysis toolboxes, such as EEGLAB.

In addition to SOUND, we discussed the DDWiener-based approaches to tackle EEG and MEG noise. As a proof of concept, we showed how DDWiener can be utilized in finding the trial-specific noise levels. According to visual inspection, DDWiener performed well. We did not, however, compare the performance of this approach to a related method, robust averaging, implemented in the SPM toolbox.

\section{Conclusion}

We introduced a method called SOUND that robustly identifies and suppresses noise and artifacts in EEG. We showed mathematically that, when assuming diagonal noise structure, SOUND provides an optimal estimate for the noiseless EEG/MEG signals, with minimum MSE. Furthermore, through simulations, we showed that SOUND is to some extent robust against the violation of this assumption.

SOUND is completely automatic up to defining the regularization parameter. We showed a strategy to define this parameter from the data. In addition to SOUND, we showed how general Wiener estimators can be used in a data-driven fashion to clean the measured multi-sensor and multi-trial data. The suggested methods provide quantitative, objective, and efficient ways to preprocess and clean the gathered data. We validated the performance of the presented methods in practice by analyzing several real-life datasets.

Along with this publication, we provide a MATLAB demo package that demonstrates how the presented methods can be applied in practice. The description of the package and the download link can be found in the Supplementary material II. In the future, we are willing to help to include these techniques to some freeware MATLAB toolbox, such as EEGLAB.

\section{Acknowledgments}

We thank Prof. Jukka Sarvas, Prof. Lauri Parkkonen, Dr. Matti Stenroos, and Dr. Jaakko Nieminen for valuable suggestions regarding the manuscript. In addition, we would like to thank Prof. Sarvas for providing tools for the MATLAB demo package. This study was supported by the Academy of Finland (Grant No. 283105), the Finnish Cultural Foundation (Grant No. 00150064, 00161149, 00140634, and 00160630), the Foundation for Aalto University Science and Technology, the Kymenlaakso Regional fund of the Finnish Cultural Foundation (35162142), and the Finnish Brain Foundation.

Some datasets used for this study were downloaded from the HeadIT Data Repository (http://www.headit.org), supported by grants to the HeadIT (R01-MH084819) and EEGLAB (5-R01-NS047293-08) funded by the National Institutes of Health, U.S.A.

\section{Appendices.}

\section{A. Wiener estimation for cleaning noise in multi-sensor data}

Here, we show that SOUND corresponds to Wiener estimation of the noiseless EEG/MEG signals. We also explain how the data-driven version of the Wiener estimator (Eq. (8)) is obtained, and that this approximation corresponds to Sensor Noise Suppression (SNS) (De Cheveigné and Simon, 2008).

We aim at estimating $\overline{\mathbf{Y}}$ from the noisy data, expressed in Eq. (1), assuming that $\mathbf{N}$ is uncorrelated with $\overline{\mathbf{Y}}$ and that the correlation matrix of $\mathbf{N}$ is diagonal. Again, we wish to find an estimate for the signal in sensor $s$ using the data recorded by all the other sensors. If we know the correlation matrix of the data, $\mathbf{C}=\operatorname{corr}(\mathbf{Y})$, and the cross-correlation vector $\mathbf{r}_{\neq s, s}$ between $\mathbf{Y}_{\neq s}$ and $\overline{\mathbf{y}}_{s}$, we can use Wiener estimation (or filtering) (Hayes, 2009) to find an estimate for the noiseless data in the sensor $s$ by

$$
\begin{aligned}
& \widehat{\mathbf{y}}_{s}^{\text {Wiener }}=\left(\widehat{\mathbf{w}}_{s}\right)^{\mathrm{T}} \mathbf{Y}_{\neq s} \\
& \widehat{\mathbf{w}}_{s}=\left(\mathbf{C}_{\neq s, \neq s}\right)^{-1} \mathbf{r}_{\neq s, s},
\end{aligned}
$$

where the optimal weight vector $\widehat{\mathbf{w}}_{s}$ is found so that the corresponding mean-squared error, $T^{-1} \sum_{t=1}^{T}\left(\bar{y}_{s, t}-\left(\widehat{\mathbf{w}}_{s}\right)^{\mathrm{T}} \mathbf{y}_{\neq s, t}\right)^{2}$, has been minimized. Wiener estimation is a robust method against small errors in $\widehat{\mathbf{w}}_{s}$ since the mean-square-error increases only in proportion to the square of the deviants from the optimal weights.

For the measured data described in Eq. (1), the data correlation matrix is found to be $\mathbf{C} \approx T^{-1}(\mathbf{L} \mathbf{J}+\mathbf{N})(\mathbf{L} \mathbf{J}+\mathbf{N})^{\mathrm{T}} \approx \mathbf{L} \boldsymbol{\Gamma} \mathbf{L}^{\mathrm{T}}+\boldsymbol{\Sigma}$, $\boldsymbol{\Gamma}$ and $\boldsymbol{\Sigma}$ being the source and noise correlation matrices, respectively. The corresponding cross-correlation vector between $\mathbf{Y}$ and $\overline{\mathbf{y}}_{s}$ can be written as $\mathbf{r}_{s, s} \approx T^{-1}(\mathbf{L J}+\mathbf{N})\left(\mathbf{1}_{s} \mathbf{J}\right)^{\mathrm{T}} \approx \mathbf{L} \boldsymbol{\Gamma} \mathbf{1}_{s}^{\mathrm{T}}$. Since the expectation values of the noise and the recorded data are assumed zero, their correlation matrices are identical with the corresponding covariance matrices. In practice, the source correlation matrix is commonly not known, and hence, the sources are 
assumed independent and identically distributed (i.i.d.), leading to $\boldsymbol{\Gamma}=\lambda^{-2} \mathbf{I}$, where $\lambda^{-2}$ is the source variance. Thus,

$$
\begin{aligned}
& \mathbf{C}_{\neq s, \neq s}=\lambda^{-2} \mathbf{L}_{\neq s} \mathbf{L}_{\neq s}^{\mathrm{T}}+\boldsymbol{\Sigma}_{\neq s, \neq s} \\
& \mathbf{r}_{\neq s, s}=\lambda^{-2} \mathbf{L}_{\neq s} \mathbf{1}_{s}^{\mathrm{T}} .
\end{aligned}
$$

Substituting these identities into Eq. (A.1) yields the estimate

$$
\begin{aligned}
& \widehat{\mathbf{y}}_{s}^{\text {i.i.d. }}=\mathbf{l}_{s} \mathbf{L}_{\neq s}^{\mathrm{T}}\left(\mathbf{L}_{\neq s} \mathbf{L}_{\neq s}^{\mathrm{T}}+\lambda^{2} \boldsymbol{\Sigma}_{\neq s, \neq s}\right)^{-1} \mathbf{Y}_{\neq s} \\
& =\mathbf{1}_{s} \tilde{\mathbf{L}}_{\neq s}^{\mathrm{T}}\left(\tilde{\mathbf{L}}_{\neq s} \tilde{\mathbf{L}}_{\neq s}^{\mathrm{T}}+\lambda^{2} \mathbf{I}\right)^{-1} \tilde{\mathbf{Y}}_{\neq s},
\end{aligned}
$$

where the latter form is written in terms of whitened data (Eq. (2)).

We see that Eq. (A.3) actually corresponds to combining Eqs. (3) and (6) in the same way as used by SOUND in the iterative estimation of the noise covariance matrix. After the iterations, SOUND takes into account all the measured data and the final estimated noise covariance matrix to reconstruct cleaned EEG using Eq. (7). Thus, the final step of SOUND corresponds to building the Wiener estimator for $\overline{\mathbf{Y}}$ using all the sensor signals as the estimator input.

An alternative approach for implementing the Wiener estimator is to use the collected data samples for computing the sample correlation matrix of the data $\mathbf{C} \simeq T^{-1} \mathbf{Y Y}^{\mathrm{T}}$. Since the noise correlation matrix is diagonal, $\mathbf{r}_{\neq s, s}=\mathbf{c}_{\neq s, s}$. This can be used to simplify the expression for the weight vector in Eq. (A.1):

$\widehat{\mathbf{w}}_{s}=\left(\mathbf{C}_{\neq s, \neq s}\right)^{-1} \mathbf{c}_{\neq s, s}$.

Note that this simplification would not be possible if the $s$ th sensor was included in the input of the estimator. Substituting this approximation for $\widehat{\mathbf{w}}_{s}$ in Eq. (A.1) yields

$\widehat{\mathbf{y}}_{s}^{\text {sample }}=\mathbf{y}_{s}\left(\mathbf{Y}_{\neq s}\right)^{\mathrm{T}}\left[\mathbf{Y}_{\neq s}\left(\mathbf{Y}_{\neq s}\right)^{\mathrm{T}}\right]^{-1} \mathbf{Y}_{\neq s}$.

This approach can be seen to be data-driven since it uses only the recorded data to estimate the noiseless signals.

It is straightforward to show that Eq. (A.5) corresponds to SNS algorithm (De Cheveigné and Simon, 2008), which replaces each noisy channel by its regression on the subspace formed by the other channels. We express the $\neq s$ rows of the data in terms of singular-value decomposition (SVD), $\mathbf{Y}_{\neq s}=\mathbf{U S V}^{\mathrm{T}}$, where the columns of $\mathbf{U}$ and $\mathbf{V}$ contain the left and right singular vectors, respectively, and $\mathbf{S}$ holds the singular values on its $K$ first diagonal entries. The column vectors of $\mathbf{V}$ form a complete orthonormal basis for all the possible signals in the rows of $\mathbf{Y}$, whereas the $K$ first columns span only the subspace needed for explaining the measured signals in sensors $\neq s$.

With the help of SVD, Eq. (A.5) can be written as:

$$
\begin{aligned}
& \widehat{\mathbf{y}}_{s}^{\text {sample }}=\mathbf{y}_{s}\left(\mathbf{U S V}^{\mathrm{T}}\right)^{\mathrm{T}}\left[\mathbf{U S V}^{\mathrm{T}}\left(\mathbf{U S V}^{\mathrm{T}}\right)^{\mathrm{T}}\right]^{-1} \mathbf{U S V}^{\mathrm{T}} \\
& =\mathbf{y}_{s} \mathbf{V S}^{\mathrm{T}}\left(\mathbf{S S}^{\mathrm{T}}\right)^{-1} \mathbf{S V}^{\mathrm{T}} .
\end{aligned}
$$

If $\mathbf{S S}^{T}$ is not invertible, one may use its generalized inverse. In either case, the estimate becomes:

$\widehat{\mathbf{y}}_{s}^{\text {sample }}=\sum_{k=1}^{K}\left(\mathbf{y}_{s} \mathbf{v}_{\cdot, k}\right)\left(\mathbf{v}_{\cdot, k}\right)^{\mathrm{T}}$,

which is the mathematical expression for SNS.

\section{B. Generating the noise for the simulated data}

Here, we describe how the correlation structure of the simulated noise, with $T$ samples and $S$ sensors, was controlled. We first randomized $T$ samples of an $S$-variate Gaussian random noise vector with zero mean and identity covariance matrix and collected them in an $S \times T$ noise matrix $\varepsilon$, where each row represents the activity (waveform) of one noise source. In the sensor space, the noise sources were mixed by matrix $\mathbf{A}$, yielding the noise signals $\mathbf{N}=\mathbf{A} \boldsymbol{\varepsilon}$. If the ith column of $\mathbf{A}$ has $m$ non-zero elements, the noise signals become correlated across the corresponding $m$ channels.

Based on this reasoning, the noise was generated by

$\mathbf{N}=\mathbf{A} \boldsymbol{\varepsilon}=\left(\mathbf{B}^{(m)} \mathrm{oL}\right) \boldsymbol{\varepsilon}$,

where $\circ$ denotes the entry-wise matrix product and $\mathbf{B}^{(m)}$ is a binary matrix with $m$ elements in each column equal to 1 (the remaining elements being zero). Thus, the signals from each noise source were correlated across $m$ channels. We call $m$ the noise correlation index (NCI). When NCI $=1$, the resulting noise covariance matrix is diagonal, whereas, when NCI approaches $S$, the noise becomes correlated across an increasing number of sensors and starts to resemble neural noise with covariance matrix $\boldsymbol{\Sigma}=\mathbf{L L}^{\mathrm{T}}$. Thus, as NCI increases, the data correction becomes increasingly difficult for SOUND. For a fixed NCI $(m)$, the non-zero elements in the columns of $\mathbf{B}^{(m)}$ were randomly chosen for each simulation.

\section{Appendix C. Supplementary data}

Supplementary data related to this article can be found at https://doi.org/10.1016/j.neuroimage.2017.10.021. 


\section{References}

Ashburner, J., Barnes, G., Chen, C.-C., Daunizeau, J., Flandin, G., Friston, K., Kiebel, S. Kilner, J., Litvak, V., Moran, R., Penny, W., Razi, A., Stephan, K., Tak, S., Zeidman, P., Gitelman, D., Henson, R., Hutton, C., Glauche, V., Mattout, J., Phillips, C., 2012. SPM12 Manual. Functional Imaging Laboratory, Wellcome Trust Centre for Neuroimaging, Institute of Neurology, UCL.

Atluri, S., Frehlich, M., Mei, Y., Dominguez, L.G., Rogasch, N.C., Wong, W., Daskalakis, Z.J., Farzan, F., 2016. TMSEEG: a MATLAB-based graphical user interface for processing electrophysiological signals during transcranial magnetic stimulation. Front. Neural Circuits 10.

Baccalá, L.A., Sameshima, K., 2001. Partial directed coherence: a new concept in neural structure determination. Biol. Cybern. 84, 463-474.

Berg, P., Scherg, M., 1994. A multiple source approach to the correction of eye artifacts. Electroencephalogr. Clin. Neurophysiology 90, 229-241.

Bertero, M., De Mol, C., Pike, E.R., 1988. Linear inverse problems with discrete data: II Stability and regularisation. Inverse Probl. 4, 573-594.

Bezerianos, A., Laskaris, N., Fotopoulos, S., Papathanasopoulos, P., 1995. Data dependent weighted averages for recording of evoked potential signals. Electroencephalogr. Clin. Neurophysiology/Evoked Potentials Sect. 96, 468-471.

Bigdely-Shamlo, N., Mullen, T., Kothe, C., Su, K.-M., Robbins, K.A., 2015. The PREP pipeline: standardized preprocessing for large-scale EEG analysis. Front. Neuroinformatics 9, 16 .

Bresnahan, S.M., Barry, R.J., 2002. Specificity of quantitative EEG analysis in adults with attention deficit hyperactivity disorder. Psychiatry Res. 112, 133-144.

Buzsaki, G., Draguhn, A., 2004. Neuronal oscillations in cortical networks. Science 304, 1926-1929.

Campbell, N.A., 1980. Robust procedures in multivariate analysis I: robust covariance estimation. Appl. Stat. 231-237.

Casarotto, S., Canali, P., Rosanova, M., Pigorini, A., Fecchio, M., Mariotti, M., Lucca, A. Colombo, C., Benedetti, F., Massimini, M., 2013. Assessing the effects of electroconvulsive therapy on cortical excitability by means of transcranial magnetic stimulation and electroencephalography. Brain Topogr. 26, 326-337.

Crouzeix, A., Yvert, B., Bertrand, O., Pernier, J., 1999. An evaluation of dipole reconstruction accuracy with spherical and realistic head models in MEG. Clin. Neurophysiol. 110, 2176-2188.

Davila, C.E., Mobin, M.S., 1992. Weighted averaging of evoked potentials. IEEE Trans. Biomed. Eng. 39, 338-345.

De Cheveigné, A., Simon, J.Z., 2008. Sensor noise suppression. J. Neurosci. Methods 168, 195-202.

Delorme, A., Makeig, S., 2004. EEGLAB: an open source toolbox for analysis of single-trial EEG dynamics including independent component analysis. J. Neurosci. Methods 134 9-21.

Delorme, A., Mullen, T., Kothe, C., Acar, Z.A., Bigdely-Shamlo, N., Vankov, A., Makeig, S., 2011. EEGLAB, SIFT, NFT, BCILAB, and ERICA: new tools for advanced EEG processing. Comput. Intell. Neurosci. 2011, 10.

Dominguez, L.G., Radhu, N., Farzan, F., Daskalakis, Z.J., 2014. Characterizing long interval cortical inhibition over the time-frequency domain. PloS One 9, e92354.

Engemann, D.A., Gramfort, A., 2015. Automated model selection in covariance estimation and spatial whitening of MEG and EEG signals. NeuroImage 108, 328-342.

Ferree, T.C., Luu, P., Russell, G.S., Tucker, D.M., 2001. Scalp electrode impedance, infection risk, and EEG data quality. Clin. Neurophysiol. 112, 536-544.

Ferreri, F., Pasqualetti, P., Määttä, S., Ponzo, D., Ferrarelli, F., Tononi, G., Mervaala, E. Miniussi, C., Rossini, P.M., 2011. Human brain connectivity during single and paired pulse transcranial magnetic stimulation. NeuroImage 54, 90-102.

Fischl, B., 2012. FreeSurfer. NeuroImage 62, 774-781.

Foster, M., 1961. An application of the Wiener-Kolmogorov smoothing theory to matrix inversion. J. Soc. Industrial Appl. Math. 9, 387-392.

Goncharova, I.I., McFarland, D.J., Vaughan, T.M., Wolpaw, J.R., 2003. EMG contamination of EEG: spectral and topographical characteristics. Clin. Neurophysiol. 114, 1580-1593.

Gramfort, A., Luessi, M., Larson, E., Engemann, D.A., Strohmeier, D., Brodbeck, C., Parkkonen, L., Hämäläinen, M.S., 2014. MNE software for processing MEG and EEG data. NeuroImage 86, 446-460.

Hämäläinen, M., Hari, R., Ilmoniemi, R.J., Knuutila, J., Lounasmaa, O.V., 1993. Magnetoencephalography - theory, instrumentation, and applications to noninvasive studies of the working human brain. Rev. Mod. Phys. 65, 413.

Hauk, O., 2004. Keep it simple: a case for using classical minimum norm estimation in the analysis of EEG and MEG data. NeuroImage 21, 1612-1621.

Hauk, O., Pulvermüller, F., 2004. Neurophysiological distinction of action words in the fronto-central cortex. Hum. Brain Mapp. 21, 191-201.

Hayes, M.H., 2009. Statistical Digital Signal Processing and Modeling. John Wiley \& Sons.

Herrmann, C.S., Demiralp, T., 2005. Human EEG gamma oscillations in neuropsychiatric disorders. Clin. Neurophysiol. 116, 2719-2733.

Hong, L.E., Summerfelt, A., McMahon, R., Adami, H., Francis, G., Elliott, A., Buchanan, R.W., Thaker, G.K., 2004. Evoked gamma band synchronization and the liability for schizophrenia. Schizophrenia Res. 70, 293-302.

Huber, P.J., 2011. Robust statistics. In: International Encyclopedia of Statistical Science. Springer, pp. 1248-1251.

Ikeda, S., Toyama, K., 2000. Independent component analysis for noisy data-MEG data analysis. Neural Netw. 13, 1063-1074.

Ilmoniemi, R.J., Hernandez-Pavon, J.C., Mäkelä, N.N., Metsomaa, J., Mutanen, T.P., Stenroos, M., Sarvas, J., 2015. Dealing with artifacts in TMS-evoked EEG. In: Engineering in Medicine and Biology Society (EMBC), 2015 37th Annual International Conference of the IEEE. IEEE, pp. 230-233.
Ilmoniemi, R.J., Kičić, D., 2010. Methodology for combined TMS and EEG. Brain Topogr. 22, 233-248.

Ilmoniemi, R.J., Virtanen, J., Ruohonen, J., Karhu, J., Aronen, H.J., Katila, T., 1997. Neuronal responses to magnetic stimulation reveal cortical reactivity and connectivity. NeuroReport 8, 3537-3540.

Jas, M., Engemann, D.A., Bekhti, Y., Raimondo, F., Gramfort, A., 2017. Autoreject: automated artifact rejection for MEG and EEG data. NeuroImage 159, 417-429.

Jensen, O., Colgin, L.L., 2007. Cross-frequency coupling between neuronal oscillations. Trends Cognitive Sci. 11, 267-269.

John, M.S., Dimitrijevic, A., Picton, T.W., 2001. Weighted averaging of steady-state responses. Clin. Neurophysiol. 112, 555-562.

Julkunen, P., Jauhiainen, A.M., Könönen, M., Pääkkönen, A., Karhu, J., Soininen, H. 2011. Combining transcranial magnetic stimulation and electroencephalography may contribute to assess the severity of Alzheimer's disease. Int. J. Alzheimer's Dis. 2011.

Kaipio, J., Somersalo, E., 2006. Statistical and Computational Inverse Problems. Springer Science \& Business Media.

Kaukoranta, E., Hämäläinen, M., Sarvas, J., Hari, R., 1986. Mixed and sensory nerve stimulations activate different cytoarchitectonic areas in the human primary somatosensory cortex SI. Exp. Brain Res. 63, 60-66.

Khan, J.A., Van Aelst, S., Zamar, R.H., 2007. Robust linear model selection based on least angle regression. J. Am. Stat. Assoc. 102, 1289-1299.

Komssi, S., Aronen, H.J., Huttunen, J., Kesäniemi, M., Soinne, L., Nikouline, V.V., Ollikainen, M., Roine, R.O., Karhu, J., Savolainen, S., Ilmoniemi, R.J., 2002. Ipsi- and contralateral EEG reactions to transcranial magnetic stimulation. Clin. Neurophysiol. $113,175-184$.

Komssi, S., Huttunen, J., Aronen, H.J., Ilmoniemi, R.J., 2004. EEG minimum-norm estimation compared with MEG dipole fitting in the localization of somatosensory sources at S1. Clin. Neurophysiol. 115, 534-542.

Korhonen, R.J., Hernandez-Pavon, J.C., Metsomaa, J., Mäki, H., Ilmoniemi, R.J., Sarvas, J., 2011. Removal of large muscle artifacts from transcranial magnetic stimulation-evoked EEG by independent component analysis. Med. Biol. Eng. Comput. 49, 397-407.

Ledoit, O., Wolf, M., 2004. A well-conditioned estimator for large-dimensional covariance matrices. J. Multivar. Analysis 88, 365-411.

Lin, F.-H., Belliveau, J.W., Dale, A.M., Hämäläinen, M.S., 2006. Distributed current estimates using cortical orientation constraints. Hum. Brain Mapp. 27, 1-13.

Litvak, V., Komssi, S., Scherg, M., Hoechstetter, K., Classen, J., Zaaroor, M., Pratt, H., Kahkonen, S., 2007. Artifact correction and source analysis of early electroencephalographic responses evoked by transcranial magnetic stimulation over primary motor cortex. NeuroImage 37, 56-70.

Mäki, H., Ilmoniemi, R.J., 2011. Projecting out muscle artifacts from TMS-evoked EEG. NeuroImage 54, 2706-2710.

Maronna, R.A., Martin, R.D., Yohai, V.J., 2006. Robust Statistics. John Wiley \& Sons.

Michel, C.M., Murray, M.M., Lantz, G., Gonzalez, S., Spinelli, L., de Peralta, R.G., 2004 EEG source imaging. Clin. Neurophysiol. 115, 2195-2222.

Moiseev, A., Gaspar, J.M., Schneider, J.A., Herdman, A.T., 2011. Application of multisource minimum variance beamformers for reconstruction of correlated neural activity. NeuroImage 58, 481-496.

Monto, S., Palva, S., Voipio, J., Palva, J.M., 2008. Very slow EEG fluctuations predict the dynamics of stimulus detection and oscillation amplitudes in humans. J. Neurosci. 28, 8268-8272.

Moratti, S., Keil, A., 2005. Cortical activation during Pavlovian fear conditioning depends on heart rate response patterns: an MEG study. Cognitive Brain Res. 25, 459-471.

Mosher, J.C., Hamalainen, M.S., Pantazis, D., Hui, H.B., Burgess, R.C., Leahy, R.M., 2009. Generalized sidelobe canceller for magnetoencephalography arrays. In: Biomedical Imaging: from Nano to Macro, 2009. ISBI'09. IEEE, pp. 149-152. IEEE International Symposium on.

Mutanen, T.P., Kukkonen, M., Nieminen, J.O., Stenroos, M., Sarvas, J., Ilmoniemi, R.J., 2016. Recovering TMS-evoked EEG responses masked by muscle artifacts. NeuroImage 139, 157-166.

Nguyen, T.-D., Welsch, R.E., 2010. Outlier detection and robust covariance estimation using mathematical programming. Adv. Data Analysis Classif. 4, 301-334.

Nieminen, J.O., Gosseries, O., Massimini, M., Saad, E., Sheldon, A.D., Boly, M., Siclari, F., Postle, B.R., Tononi, G., 2016. Consciousness and cortical responsiveness: a withinstate study during non-rapid eye movement sleep. Sci. Rep. 6, 30932

Niso, G., Rogers, C., Moreau, J.T., Chen, L.-Y., Madjar, C., Das, S., Bock, E., Tadel, F., Evans, A.C., Jolicoeur, P., et al., 2016. OMEGA: the open MEG archive. NeuroImage 124, 1182-1187.

Nolan, H., Whelan, R., Reilly, R., 2010. FASTER: fully automated statistical thresholding for EEG artifact rejection. J. Neurosci. Methods 192, 152-162.

Numminen, J., Ahlfors, S., Ilmoniemi, R., Montonen, J., Nenonen, J., 1995. Transformation of multichannel magnetocardiographic signals to standard grid form. IEEE Trans. Biomed. Eng. 42, 72-78.

Onton, J., Westerfield, M., Townsend, J., Makeig, S., 2006. Imaging human EEG dynamics using independent component analysis. Neurosci. Biobehav. Rev. 30, 808-822.

Parra, L.C., Spence, C.D., Gerson, A.D., Sajda, P., 2005. Recipes for the linear analysis of EEG. NeuroImage 28, 326-341.

Perrin, F., Pernier, J., Bertrand, O., Echallier, J.F., 1989. Spherical splines for scalp potential and current density mapping. Electroencephalogr. Clin. Neurophysiology 72, 184-187.

Picton, T.W., Bentin, S., Berg, P., Donchin, E., Hillyard, S.A., Johnson, R., Miller, G.A., Ritter, W., Ruchkin, D.S., Rugg, M.D., Taylor, M.J., 2000. Guidelines for using human event-related potentials to study cognition: recording standards and publication criteria. Psychophysiology 37, 127-152. 
Portin, K., Vanni, S., Virsu, V., Hari, R., 1999. Stronger occipital cortical activation to lower than upper visual field stimuli Neuromagnetic recordings. Exp. Brain Res. 124 287-294.

Rousseeuw, P.J., 1984. Least median of squares regression. J. Am. Stat. Assoc. 79, $871-880$.

Shattuck, D.W., Leahy, R.M., 2002. BrainSuite: an automated cortical surface identification tool. Med. Image Anal. 6, 129-142.

Stenroos, M., Sarvas, J., 2012. Bioelectromagnetic forward problem: isolated source approach revis(it)ed. Phys. Med. Biol. 57, 3517-3535.

Styliadis, C., Ioannides, A.A., Bamidis, P.D., Papadelis, C., 2014. Amygdala responses to valence and its interaction by arousal revealed by MEG. Int. J. Psychophysiol. 93 , $121-133$.

Taulu, S., Kajola, M., 2005. Presentation of electromagnetic multichannel data: the signal space separation method. J. Appl. Phys. 97, 124905.

Taulu, S., Kajola, M., Simola, J., 2004. Suppression of interference and artifacts by the signal space separation method. Brain Topogr. 16, 269-275.

Tewarie, P., Hillebrand, A., Schoonheim, M., Van Dijk, B.W., Geurts, J.J.G., Barkhof, F. Polman, C.H., Stam, C.J., 2014. Functional brain network analysis using minimum spanning trees in Multiple Sclerosis: an MEG source-space study. NeuroImage 88, 308-318.

Uusitalo, M.A., Ilmoniemi, R.J., 1997. Signal-space projection method for separating MEG or EEG into components. Med. Biol. Eng. Comput. 35, 135-140.

Uutela, K., Taulu, S., Hämäläinen, M., 2001. Detecting and correcting for head movements in neuromagnetic measurements. NeuroImage 14, 1424-1431.

Van der Meer, M.L., Tewarie, P., Schoonheim, M.M., Douw, L., Barkhof, F., Polman, C.H., Stam, C.J., Hillebrand, A., 2013. Cognition in MS correlates with resting-state

oscillatory brain activity: an explorative MEG source-space study. NeuroImage Clin. 2, 727-734.

Vigário, R.N., 1997. Extraction of ocular artefacts from EEG using independent component analysis. Electroencephalogr. Clin. Neurophysiology 103, 395-404.

Vrba, J., Robinson, S.E., 2001. Signal processing in magnetoencephalography. Methods 25, 249-271.

Whitham, E.M., Pope, K.J., Fitzgibbon, S.P., Lewis, T., Clark, C.R., Loveless, S., Broberg, M., Wallace, A., DeLosAngeles, D., Lillie, P., Hardy, A., Fronsko, R. Pulbrook, A., Willoughby, J.O., 2007. Scalp electrical recording during paralysis: quantitative evidence that EEG frequencies above $20 \mathrm{~Hz}$ are contaminated by EMG. Clin. Neurophysiol. 118, 1877-1888. 